\title{
Current Molecular Therapeutic Agents and Drug Candidates for Mycobacterium abscessus
}

\author{
Nguyen Thanh Quang and Jichan Jang *
}

Molecular Mechanisms of Antibiotics, Division of Life Science, Department of Bio and Medical Big Data (BK21 Four Program), Research Institute of Life Science, Gyeongsang National University, Jinju, South Korea

Mycobacterium abscessus has been recognised as a dreadful respiratory pathogen among the non-tuberculous mycobacteria (NTM) because of misdiagnosis, prolonged therapy with poor treatment outcomes and a high cost. This pathogen also shows extremely high antimicrobial resistance against current antibiotics, including the antituberculosis agents. Therefore, current chemotherapies require a long curative period and the clinical outcomes are not satisfactory. Thus, there is an urgent need for discovering and developing novel, more effective anti- $M$. abscessus drugs. In this review, we sum the effectiveness of the current anti-M. abscessus drugs and drug candidates. Furthermore,

OPEN ACCESS

Edited by:

Hendra Gunosewoyo,

Curtin University, Australia

Reviewed by:

Mario Salmeri,

University of Catania, Italy

Matt Johansen,

University of Technology Sydney,

Australia

${ }^{*}$ Correspondence:

Jichan Jang

jichanjang@gnu.ac.kr

Specialty section:

This article was submitted to Experimental Pharmacology and

Drug Discovery,

a section of the journal

Frontiers in Pharmacology

Received: 14 June 2021 Accepted: 03 August 2021

Published: 30 August 2021

Citation:

Quang NT and Jang J (2021) Current Molecular Therapeutic Agents and

Drug Candidates for

Mycobacterium abscessus.

Front. Pharmacol. 12:724725.

doi: $10.3389 /$ fphar.2021.724725 we describe the shortcomings and difficulties associated with $M$. abscessus drug discovery and development.

Keywords: Mycobacterium abscessus, anti-M. abscessus, antibiotics, drug resistance, drug discovery

\section{INTRODUCTION}

A steady increase in the morbidity and mortality rates of non-tuberculous mycobacteria (NTM) has been noted worldwide; surpassing the numbers of tuberculosis (TB), NTM is becoming a new global health concern. NTM has been noted to increasingly cause pulmonary-associated morbidity and mortality in the United States, although NTM lung disease remains uncommon in the general United States population (estimated in 40 cases/100,000 persons). However, NTM is highly prevalent among adults and children with cystic fibrosis (CF). According to an analytical study in the epidemiology of pulmonary NTM sputum positivity from 16,153 patients with CF in the United States, 20\% $(3,211)$ had a pathogenic NTM species. Among the NTM, 61\% had Mycobacterium avium complex (MAC) and 39\% had M. abscessus (hereafter Mab) (Adjemian et al., 2018). Among the NTM, Mab is described as an opportunistic, emerging, non-tuberculous, and saprophytic Mycobacterium found commonly in soil and water systems (Falkinham, 2013; Morimoto et al., 2018). However, this organisms can cause nosocomial outbreaks and pseudooutbreaks due to contaminated materials (Roux et al., 2016). For instance, NTM has been detected in hospital ice machines, water-cooling systems and haemodialysis unit water supplies (Ratnatunga et al., 2020). Furthermore, recent studies demonstrated that NTM species, including Mab, have been identified in showerhead biofilms, which have become the primary source of NTM exposure to humans with a high organism density (Gebert et al., 2018).

The clinical spectrum of Mab has been broadly categorised as a pulmonary and extrapulmonary disease. Mab is the most common cause of rapidly growing mycobacteria (RGM) pulmonary infections, particularly in immunocompromised patients, such as those with $\mathrm{CF}$, human immunodeficiency virus-positive status, chronic obstructive pulmonary disease, and bronchiectasis (Bryant et al., 2013; Mougari et al., 2016; Skolnik et al., 2016; Wassilew et al., 
2016; Andrew et al., 2019; Stephenson et al., 2019; Johansen et al., 2020b). Furthermore, according to recent studies, most Mab pulmonary disease cases have been identified in healthy older adults with no history of smoking but who have lung airway abnormalities (Ryan and Byrd, 2018). The pulmonary Mab disease is known as a chronic and incurable disease and it requires treatment with parenteral antibiotics for 2-4 months followed by long-term macrolide-based antibiotic therapy (Griffith et al., 2007). In contrast, Mab extrapulmonary infection can occur many sites such as skin, soft tissue, and bone infections after medical procedures or traumatic injuries (Wi, 2019). The recommended duration of therapy for extrapulmonary infection is usually a total of 4-6 months of antibiotic treatment with an initial combination of parenteral antibiotics for at least 2 weeks with a high success rate (Griffith et al., 2007).

The Mab group is known to consist of three subspecies, namely, Mab subsp. abscessus, Mab subsp. massiliense and Mab subsp. bolletii (Minias et al., 2020). Each subspecies is different in terms of clinical outcomes and typical antimicrobial susceptibility profile (Blauwendraat et al., 2012; Harada et al., 2012; Shin et al., 2013; Jeong et al., 2017; Abate et al., 2019). The macrolide (azithromycin and clarithromycin)resistant ability of Mab subsp. abscessus is induced by an adaptive resistance mechanism using the inducible ribosomal methylase gene erm (41) (Stout and Floto, 2012; Rubio et al., 2015; Christianson et al., 2016; Abate et al., 2019). Therefore, great caution is required when using macrolide to treat $M a b$ infections (Maurer et al., 2014). Although Mab subsp. massiliense is the latest subspecies in this group; it is more widely distributed than other subspecies (Sabin et al., 2017). This subspecies has more favourable clinical outcomes than the other two members because it lacks the functional erm gene (Koh et al., 2011; Shallom et al., 2013; Park et al., 2017; Abate et al., 2019). Recent studies have shown that the transmission source of Mab subsp. massiliense is primarily the sputum with high organism loads rather than an environmental source, and most recent outbreaks occur in transplant centres, serving patients with CF (Sabin et al., 2017). Meanwhile, Mab subsp. bolletii is the rarest among the three subspecies, and it is also resistant to clarithromycin and kanamycin (Sabin et al., 2017).

Based on the American Thoracic Society/Infectious Diseases Society of America recommendation, Mab therapy comprises intravenous injection of amikacin with cefoxitin or imipenem and an oral dose of macrolide (Jeon et al., 2009). However, Mab is resistant to many antibiotics, including the above regimen, thus making it difficult to cure. Half of all patients were cured when this regimen was used, but most cases relapsed and died (Kim et al., 2019; McNeil et al., 2020). This poor success rate is mainly caused by rapidly emerging drug resistance due to their natural and acquired multidrug resistance to antibiotics. Even first-line anti-TB drugs, such as isoniazid and rifampicin, are not active against Mab. This resistance mechanism presumably involves the efflux pump mechanism, drug inactivation by ADPribosyltransferase and erythromycin resistance genes. Thus, no antibiotic class or regimen is effective for long-term sputum smear conversion in pulmonary Mab infections. Novel alternative drugs to the existing regimen are therefore required. Significant efforts to develop novel anti-TB drugs have been launched, but these drugs are deemed less potent against RGM, particularly for Mab (Chopra et al., 2011). For instance, Telacebec (Q203), a drug candidate in phase II clinical trial for Mycobacterium tuberculosis $(M t b)$, targets the QcrB in cytochrome $b c_{1}$ complex, which failed to inhibit the Mab growth at the 10,000-fold minimum inhibitory concentration required to inhibit the growth of $50 \%$ of $M t b$ (MIC 50 ) (Sorayah et al., 2019). $M a b$ drug pipelines are rarely populated and are primarily focused on the reformulation of approved antibiotics or repurposing. According to records from NIH ClinicalTrials. gov, there are only six recruiting, three completed, one terminated and one unknown clinical trial to evaluate drug efficacy. However, these clinical trials have been conducted mainly using current antibiotics in different types of drug administration, such as inhalation, new drug encapsulation with biocompatible liposomes and new drug combinations (Table 1). There are currently no FDA-approved antibiotics to treat Mab pulmonary disease (Maggioncalda et al., 2020). Although discovering novel drugs against $M a b$ is receiving much scientific attention, our current endeavours remain insufficient. Thus, discovering new alternative compounds for $M a b$ infection treatment are urgently needed. In this context, many different screens have been recently performed, such as reporter-based assays, resazurin-based microplate assay and image-based phenotypic screens (Gupta et al., 2017; Jeong et al., 2018; Richter et al., 2018; Kim et al., 2019; Malin et al., 2019; Hanh et al., 2020a, 2020b). However, there is still a poor, promising new chemical lead waiting for clinical trials and market release (Hanh et al., 2020a). This may be because of the intrinsic drug-resistant mechanism that generates the low hit rate for compounds targeting Mab (Malin et al., 2019). The hit rate of $M a b$ screens is extremely lower than the results obtained for $M t b$ screens (Malin et al., 2019). In addition, recent Mab drug screens have used preselected compounds and compound libraries reconstructed using compounds with known antimycobacterial or antibacterial properties (Malin et al., 2019). Therefore, new libraries designed with expanded chemical diversity should be prepared to identify new chemical entities. Lastly, more reliable cell-based screening that can mimic the Mab-infected host environment is strongly required.

\section{Effectiveness and Limitation of Current Anti-Mab Drugs and Drug Candidates}

Several clinical guidelines are recommended and in use (Haworth et al., 2017; Daley et al., 2020). However, these recommendations are based on a lack of high-level clinical evidence, often the clinical opinion or clinical case report (Lipman et al., 2021). Mab infection normally requires 18 months of long-term therapy with multidrug. The conventional regimen includes macrolide as a key drug in combination with two parenteral agents, often amikacin with a $\beta$-lactam-imipenem or cefoxitin for the initial phase (Haworth et al., 2017). This therapy should be given for at least 2-4 months, followed by oral macrolide based therapy (Victoria et al., 2021). The 2017 British Thoracic Society 
TABLE 1 | Current clinical trials evaluating regimens for Mab treatment (ClinicalTrials.gov).

\begin{tabular}{|c|c|c|c|c|c|c|}
\hline Status & Title & Treatment & Type & $\begin{array}{c}\text { No. of } \\
\text { participant }\end{array}$ & Phase & $\begin{array}{l}\text { Estimated } \\
\text { completion date }\end{array}$ \\
\hline \multirow[t]{6}{*}{ Recruiting } & $\begin{array}{l}\text { Liposomal amikacin inhalation in Mab } \\
\text { patients }\end{array}$ & Liposomal amikacin & Observational & 400 & & March 31, 2024 \\
\hline & $\begin{array}{l}\text { Finding the optimal regimen for Mab } \\
\text { treatment }\end{array}$ & $\begin{array}{l}\text { Amikacin/Tigecycline/Imipenem/Cefoxitin/ } \\
\text { Azithromycin/Clarithromycin /Clofazimine/ } \\
\text { Ethambutol/Linezolid/co-trimoxazole/ } \\
\text { Doxycycline/Moxifloxacin/Bedaquiline/ } \\
\text { Rifabutin }\end{array}$ & Interventional & 300 & 2 and 3 & August 31, 2023 \\
\hline & $\begin{array}{l}\text { Pilot study to assess the effect of intermittent } \\
\text { iNO on the treatment of NTM lung infection in } \\
\text { CF and non-CF patients }\end{array}$ & LungFit & Interventional & 20 & N.A. & May 2022 \\
\hline & Study of Mycobacterial infections & & Observational & 1000 & & January 1, 2001 \\
\hline & $\begin{array}{l}\text { IV gallium study for patients with cystic } \\
\text { fibrosis who have NTM }\end{array}$ & Gallium nitrate & Interventional & 40 & 1 & April 30, 2023 \\
\hline & $\begin{array}{l}\text { The Italian registry of pulmonary Non- } \\
\text { tuberculous Mycobacteria }\end{array}$ & & Observational & 500 & & $\begin{array}{l}\text { December 31, } \\
2022\end{array}$ \\
\hline \multirow[t]{2}{*}{ Completed } & Inhaled nitric oxide for patients with Mab & Nitric oxide & Interventional & 9 & 2 & April 11, 2019 \\
\hline & $\begin{array}{l}\text { Liposomal amikacin for inhalation in the } \\
\text { treatment of Mab lung disease }\end{array}$ & LAl plus multi-drug regimen & Interventional & 30 & 2 & $\begin{array}{l}\text { December 31, } \\
2019\end{array}$ \\
\hline Terminated & $\begin{array}{l}\text { Trial of inhaled molgramostim in CF subjects } \\
\text { with NTM infection }\end{array}$ & $\begin{array}{l}\text { Molgramostim nebulizer solution and eFlow }{ }^{\circledR} \\
\text { Nebulizer System (PARI Pharma GmbH) }\end{array}$ & Interventional & 14 & 2 & October 2, 2020 \\
\hline
\end{tabular}

LungFit: experimental device that produces nitric oxide from the ambient air.

INO, inhaled nitric oxide; $N$, intravenous; LAl, liposomal amikacin for inhalation; NA, not applicable.

guidelines recommended a new regimen for the initial phase as an initial phase of at least 4 weeks course of intravenous amikacin, tigecycline, and imipenem administration with a macrolide. For the continuation phase, nebulized amikacin and an oral macrolide in combination with one to three of the following oral antibiotics guided: linezolid, clofazimine, minocycline cotrimoxazole, and moxifloxacin (Haworth et al., 2017). The main goal of this guideline is 12-month sputum culture conversion. However, recurrence and several adverse effects are frequent, making this outcome unrealistic for many patients (Lyu et al., 2011; Koh et al., 2014). Here, we present current anti-Mab drugs, problems and provide an update on recent developments in the $M a b$ drug-development pipeline.

\section{Macrolide}

Treatments for Mab infection require a long-term course with multiple antibiotics. Among them, the second-generation macrolides clarithromycin and azithromycin are cornerstone components of Mab treatment (Griffith et al., 2007). Although macrolides are the main agents of Mab multidrug therapy, the treatment success rates are poor owing to macrolide-resistant strains. The primary macrolide-acquired resistance normally occurs via a specific position on the 23S rRNA $r r l$ gene; the 2058 (A2058G/C/T) or 2059 (A2059G/C) mutation in $r r l$ provides resistance to clarithromycin (de Carvalho et al., 2018; Wu et al., 2018; DanielWayman et al., 2019; Richard et al., 2020). Furthermore, Mab. subsp. abscessus and Mab. subsp. bolletii contain intrinsic resistance against macrolides mediated by erm (41). Upon exposure to the macrolide, $M a b$ induces the expression of the erm (41) gene, and its gene product transfers methyl groups to adenine in the peptidyl region of 23S rRNA, consequently modifying the binding site of clarithromycin on the 23S ribosomal RNA (Stout and Floto, 2012; de Carvalho et al., 2018) (Figure 1). Exposure to subinhibitory concentrations of clarithromycin induces transcription of erm (41) by induction of a transcriptional regulator whiB7 (Pryjma et al., 2017). However, Nash et al. reported some clinical isolates that contain non-functional erm (41). These strains did not show macrolide-inducible resistance under extended incubation with clarithromycin. Instead, the authors revealed that the strains comprise loss-of-function on the erm (41) gene with a T-to-C substitution at position 28 (C28 sequevar), resulting in an amino acid substitution from Trp to Arg at codon 10 (Nash et al., 2009; Brown-Elliott et al., 2015). Therefore, it would be worthy to sequence the erm (41) gene for prediction of macrolide susceptibility. In contrast, isolates of Mab subsp. massiliense carry a non-functional erm (41) gene that contains $397 \mathrm{bp}$ deletion, including position $28 \mathrm{~T}$, consequently do not show inducible-macrolide resistance, albeit it showed macrolide resistance due to $r r l$ mutants (Brown-Elliott et al., 2015).

To overcome the existing macrolide resistance mechanisms, new drug discovery and drug design have been evaluated. For instance, a novel class of macrolide antibiotics named "macrolones" comprising a macrocyclic moiety, linker, and either free or esterified quinolone group showed excellent antibacterial activity towards erythromycin-resistant grampositive and gram-negative bacterial strains (Čipčić Paljetak et al., 2016). Furthermore, the macrolones possess a low clearance, large volume of distribution, long half-life and possess favourable pharmacokinetic properties by accumulating in inflammatory cells, consequently complying with a once-daily dosing potential (Munić Kos et al., 2013). However, although there are considerable data available on its in vitro activity, mode of action, in vivo efficacy and its recognition as a superior compound than other known macrolides, more detailed information on the structure-associated binding mode to the ribosome is needed (Jelić and Antolović, 2016). Furthermore, its activity against Mab is yet to 


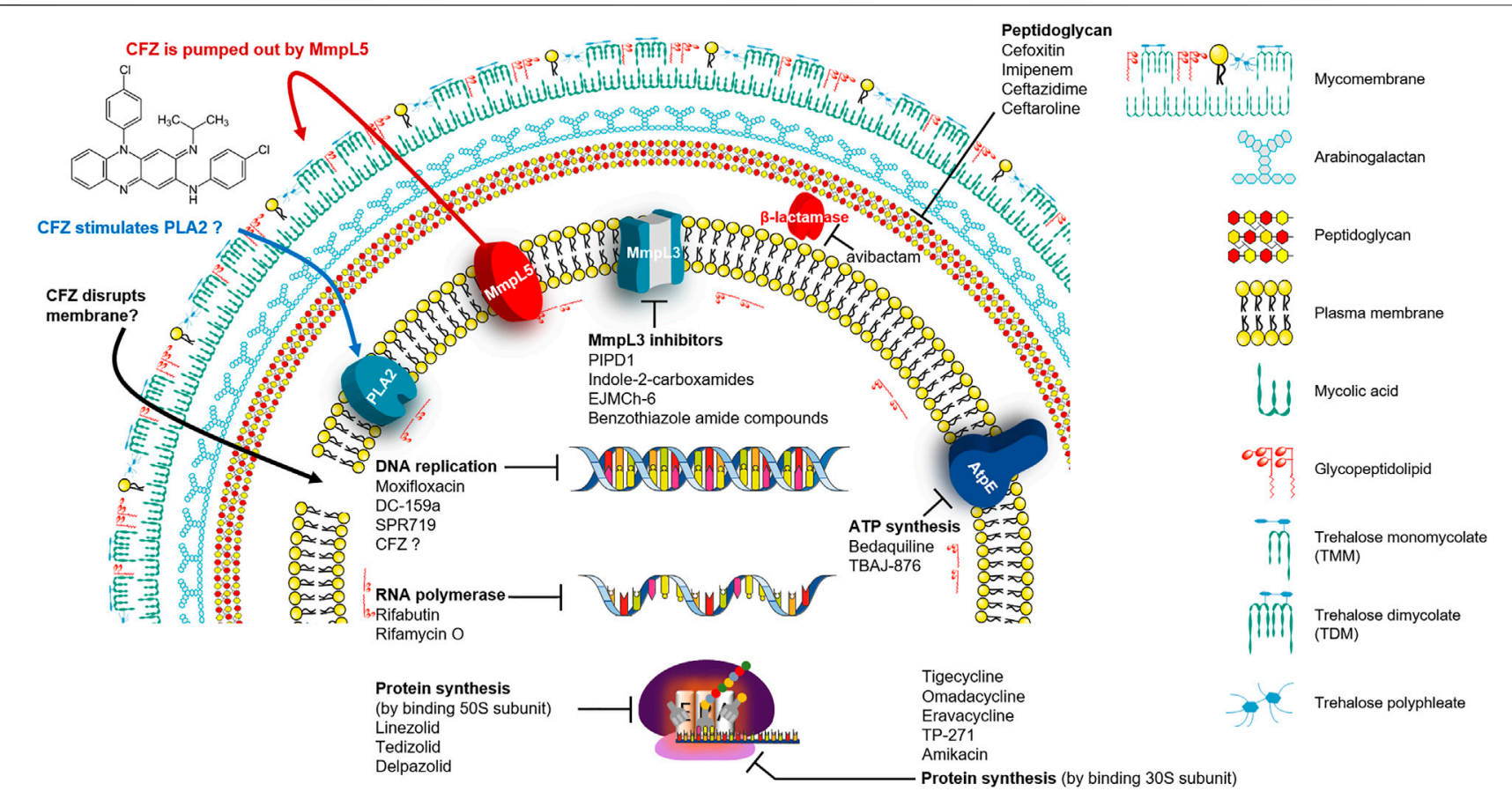

FIGURE 1 | Mechanisms of action (MOA) of anti-Mab drugs and drug candidates.

be determined. A better understanding of the macrolones in various animal models and how the macrolones in Mab impact potency is also needed.

\section{$\beta$-Lactams and $\beta$-Lactamase Inhibitor}

$\beta$-lactams are a widely-used antibiotic class, and their safety and efficacy profiles have been well-documented. $\beta$-lactams inhibit the synthesis of an essential component of the bacterial cell wall, the peptidoglycan (Hartmann et al., 1972). Peptidoglycan of the Mab contains predominantly $3 \rightarrow 3$ cross-links (64-74\%) generated by L,D-transpeptidases that are considered attractive targets for antiMab drugs. (Lavollay et al., 2011). Penicillin-binding proteins (PBPs) bind to $\beta$-lactams and this binding, in turn, interrupts the terminal transpeptidation process; consequently, it induces loss of viability and lysis of bacterial cells (Eckburg et al., 2019) (Figure 1). These have been studied extensively to treat drug-resistant $M t b$ infections, and certain $\beta$-lactam subclasses also exhibit activity against Mab. However, most of the other $\beta$-lactam antibiotics are not considered owing to their rapid hydrolysis by broad-spectrum $\beta$-lactamase (MAB_2875; BlaMab), which was reported as the major determinant of $\beta$-lactam resistance in Mab (Dubée et al., 2014; Soroka et al., 2014; Pandey et al., 2019). Only two $\beta$-lactams, such as cefoxitin and imipenem, which are relatively stable in the presence of BlaMab, showed moderate in vitro activities, and both compounds also exhibited a favourable in vivo activity in the Mabinfected zebrafish and mouse model (Table 2) (Bernut et al., 2014; Lerat et al., 2014; Moigne et al., 2020). Both drugs are currently recommended in treatment guidelines (Kozikowski et al., 2017; Pandey et al., 2019).

Interestingly, recent research on avibactam, a non- $\beta$-lactam $\beta$-lactamase inhibitor combined with $\beta$-lactam antibiotics in
$M a b$, has brought significant attention back to the re-use of $\beta$-lactams for $M a b$ treatment. Treatment with avibactam clearly showed reduced MICs of several $\beta$-lactams against Mab (Kaushik et al., 2017; Eckburg et al., 2019; Story-Roller et al., 2019). Furthermore, Dubée et al., demonstrated that knockout of the $\beta$-lactam-modifying gene (MAB_2875) restored the activity of $\beta$-lactams. Furthermore, BlaMab is inactivated by the $\beta$-lactamase inhibitor avibactam, resulting in significantly improved intramacrophagic and in vivo activity in a zebrafish model of Mab infection (Dubée et al., 2014; Lefebvre et al., 2017). Recently, the use of avibactam has gained more interest (Figure 1). The triple combination of rifabutin, imipenem and avibactam achieved five-fold killing in the numbers of intracellular survival of Mab (Le Run et al., 2018). Therefore, inhibition of functional $\beta$-lactamase activity would be crucial for the $\beta$-lactamrepurposing strategy following $M a b$ treatment. In addition, an interesting result was also reported by Pandey et al. They discovered that dual $\beta$-lactam combinations, such as that of ceftazidime with ceftaroline or ceftazidime with imipenem, showed a persistent bactericidal effect in vitro, and these combinations also showed dramatically reduced bacterial burden to near baseline levels of infection against Mabinfected THP-1 human macrophages. Again, these unexpected synergistic activities provide the possibility of re-use of a large family of $\beta$-lactam drugs as a treatment strategy against Mab infections through proper combinations (Pandey et al., 2019).

\section{Tetracycline Antibiotics}

Tetracyclines were discovered in 1945; they were known to inhibit bacterial growth by blocking the attachment of charged aminoacyl-tRNA to the A site on ribosomes, inducing failure 


\begin{tabular}{|c|c|c|c|c|}
\hline Class & Name & Chemical structure & $\mathrm{MIC}_{90}(\mathrm{mg} / \mathrm{L})$ & Efficacy \\
\hline \multirow{4}{*}{ Tetracycline } & Tigecycline & & $2-16$ & $\begin{array}{l}\text { - Increasing lifespan of Mab-infected fruit fly } \\
\text { - Proving dose-dependently effective against Mab in GM-CSF knockout mice } \\
\text { - Combination therapy showed treatment success in retrospective analysis } \\
\text { - Severe adverse effect mainly gastrointestinal distress }\end{array}$ \\
\hline & Omadacycline & & 2 & $\begin{array}{l}\text { - Tolerability is acceptable for patients with Mab disease } \\
\text { - Improved AUC/MIC than tigecycline (8-10 times) } \\
\text { - Significantly less gastrointestinal distress and fewer TEAEs }\end{array}$ \\
\hline & Eravacycline & & 1 & - Improved AUC/MIC than tigecycline (2 times) \\
\hline & TP-271 & & $0.06-0.5$ & $\begin{array}{l}\text { - In vivo efficacy has not yet tested against Mab infected animal } \\
\text { - Currently under investigation in clinical phase I }\end{array}$ \\
\hline \multirow{2}{*}{ Rifamycin } & Rifabutin & & $0.9-4.2$ & $\begin{array}{l}\text { - Good intracellular penetration/distribution } \\
\text { - Less drug-drug interaction } \\
\text { - No antagonistic effect with anti-Mab antibiotics } \\
\text { - Showing very good in vivo efficacy against Mab either in a zebrafish model and in NOD.CB17-Prkdcscid/ } \\
\text { NCrCrl mouse model }\end{array}$ \\
\hline & Rifamycin O & & $3-4.7^{\star}$ & - Good anti-Mab activity in zebrafish model of infection \\
\hline \multirow[b]{2}{*}{ Oxazolidinone } & Tedizolid & & $4-8$ & $\begin{array}{l}\text { - } 2-16 \text { fold greater in vitro activity than linezolid against Mab } \\
\text { Bacteriostatic activity }\end{array}$ \\
\hline & Delpazolid & & $1.2^{*}$ & $\begin{array}{l}\text { - Comparable efficacy to linezolid in mouse model } \\
\text { - Less myelosuppression in phase I clinical trial } \\
\text { - Currently in clinical phase II for pulmonary TB }\end{array}$ \\
\hline \multirow{2}{*}{ Diarylquinoline } & Bedaquiline & & $0.062-0.125$ & $\begin{array}{l}\text { - Good in vivo efficacy in zebrafish with less abscess and cord } \\
\text { - Significant bacterial reduction in GKO and SCID mouse model } \\
\text { - Less effectiveness in nude mouse model } \\
\text { - Doubtful result in small scale salvage therapy (4 patients) } \rightarrow \text { only one patient showed improvement of } \\
\text { clinical symptoms }\end{array}$ \\
\hline & TBAJ-876 & & $0.2-0.7$ & $\begin{array}{l}\text { - Improved tolerability and PK profiles than bedaquiline } \\
\text { - Good in vivo efficacy in mouse infection model }\end{array}$ \\
\hline
\end{tabular}


TABLE 2 | (Continued) The current state of Mab antibiotics, with compounds currently going through development. * Indicates $\mathrm{MIC}_{50}$ value.

\section{Class}

Name

Chemical structure

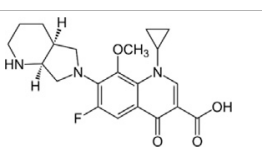

$\mathrm{MIC}_{90}(\mathrm{mg} / \mathrm{L})$

Moxifloxacin

$\prod_{0}^{\mathrm{OH}}$

$>8$

Efficacy

- Inducible mutational resistance

- Maybe used as oral drug in combination with other anti-Mab drug

- No mutation on gyrA and gyrB has been identified in Mab

DC-159a

16

- 4- to 8-fold lower MIC than other quinolones to Mab

Fluoroquinolones

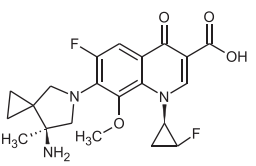

$\mathrm{H}_{3} \mathrm{C}^{\cdots} \mathrm{NH}_{2} \mathrm{H}_{3} \mathrm{C}^{-\mathrm{O}} \triangle \mathrm{F}$

Clofazimine

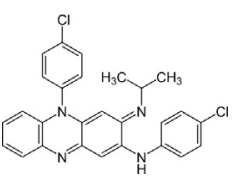

$\leq 1$

32-64

- Moderate in vitro activity

- Good in vivo efficacy in GKO mouse model of Mab infection

- Synergistic effect with AMK and CLA in in vitro combination

- Long half-life, slow metabolic elimination, high conc. in macrophage MOA is not clear

- Good in vivo efficacy in fruit fly, SCID, GKO, and GMCSF mouse

- CFZ containing regimen was revealed as effective against Mab in retrospective study

Cefoxitin

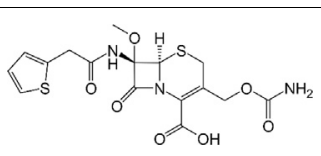

$16-64$

Imipenem

$$
\overbrace{\mathrm{O}}^{\mathrm{OH}}
$$

- Good anti-Mab activity in zebrafish model of infection

- Favorable in vivo efficacy in $\mathrm{C} 3 \mathrm{HeB} / \mathrm{FeJ}$ mouse model of Mab infection

- Synergistic activity with ceftazidime (in vitro and macrophage infection model)

$\beta$-lactams

Ceftazidime

Ceftaroline

$$
\text { 每) }
$$

$32-1024$

- Poor in vitro activity

- Synergistic activity with ceftaroline or imipenem (in vitro and macrophage infection model)

Avibactam

$\beta$-lactamase inhibitor

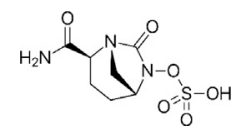

- No activity against Mab

- A non- $\beta$-lactam $\beta$-lactamase inhibitor, efficiently inhibits BlaMab.

- $\beta$-lactam combinations plus avibactam exhibited synergistic effect

- Those combination showed significant CFU reductions in the lungs of mice 
TABLE 2 | (Continued) The current state of Mab antibiotics, with compounds currently going through development. * Indicates $\mathrm{MIC}_{50}$ value.

\section{Class}

Name

Chemical structure

PIPD1

(1)

MIC $_{90}(\mathrm{mg} / \mathrm{L})$

\section{Efficacy}

$0.125-0.5$

- Excellent activity in Mab-zebrafish infection model

- Resistant mutation was identified on MAB_4508

Indole-2-
carboxamides

MmpL3 inhibitors

carboxamides

(compound 25)

\begin{tabular}{|c|c|c|}
\hline EJMCh-6 & $0.031-1$ & $\begin{array}{l}\text { - Very good activity against intracellular Mab } \\
\text { - Significant activity in zebrafish model of Mab infection }\end{array}$ \\
\hline
\end{tabular}

Benzothiazole amides

$$
\mathrm{C}_{3} \mathrm{C}_{\mathrm{O}}^{\mathrm{N}}
$$

0.5 (CRS400226)

- $0.64 \log _{10}$ bacterial load reduction with minor airway-centric inflammation in GM-CSF KO mouse

Epetraborole

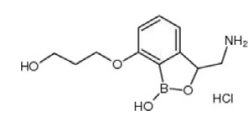

$0.1-1.35$

- Significant activity against Mab in vitro, intracellular and in zebrafish infection model

- Clinical phase II study for UTI and intra-abdominal infection was terminated due to rapi d emergence of resistant mutant

$\mathrm{AR}-12$

SPR719

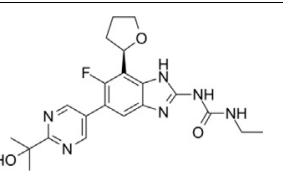

New anti-Mab

inhibitors

(2) $3.8-24.9$

Etamycin

$3.8-24.9$

- Inhibit growth of intracellular Mab

- Good activity in Mab-zebrafish infection mode

\begin{tabular}{ll}
\hline Thiostrepton & - Significant inhibition of Mab growth in vitro and in macrophages \\
& - Treated macrophages decreased proinflammatory cytokine production \\
& - Good activity in Mab-zebrafish infection model
\end{tabular}

- A phase I clinical trial has been completed

- AR-12 has received recently FDA IND-approval for cancer treatment

- Orphan drug status in Europe for selected indications

- Anti-pathogenic activity against bacteria, fungi, and viruses

- Very good in vivo efficacy in Mab infected mouse model

- Showed good in vivo efficacy result in Mab. subsp. bolletii infected SCID mouse 
of protein synthesis (Obrecht et al., 2011) (Figure 1). Usually, the changes of tetracycline efflux or ribosomal protection become resistant to tetracycline (Greer, 2006). For this reason, several tetracycline analogues have been designed and synthesised to prevent the development of resistance to tetracyclines. Although Mycobacterium smegmatis and Mtb showed low levels of intrinsic resistance to tetracycline, $\mathrm{Mab}$ is approximately 500 -fold more resistant to tetracyclines than $M$. smegmatis and $M t b$, making them unavailable for treatment (Rudra et al., 2018).

Tigecycline, the first commercially available glycylcycline, was created to bypass the critical mechanisms of bacterial resistance to tetracyclines. Changes in the tetracycline structure allow tigecycline to show good activity against tetracycline-resistant pathogens, such as penicillin-resistant Streptococcus pneumoniae, methicillin-resistant Staphylococcus aureus (MRSA) and Staphylococcus epidermidis (MRSE), and vancomycin-resistant enterococcus (VRE) species. Furthermore, tigecycline is a poor substrate of the MabTetX (MAB_1496c), tetracyclineinactivating monooxygenase, and it remains more effective than other tetracyclines analogues (Johansen et al., 2020b). Tigecycline was approved in Europe and the United States to treat complicated skin diseases and complicated intra-abdominal infections as the first drug in this class of antimicrobials (Greer, 2006). Tigecycline has in vitro activity against the Mab complex, and the minimum inhibitory concentration required to inhibit the growth of $90 \%$ of the organisms $\left(\mathrm{MIC}_{90}\right.$ ) was $2-16 \mathrm{mg} / \mathrm{L}$ (Singh et al., 2014). In the Drosophila infection model, the lifespan of Mab-infected Drosophila treated with tigecycline is much-expanded than that of Mab-infected Drosophila treated with linezolid, amikacin and cefoxitin. The combination of tigecycline and linezolid has an excellent in vivo efficacy in boosting infected fly survival and reducing Mab dissemination compared with a cocktail regimen (clarithromycin, amikacin plus cefoxitin) (Oh et al., 2014). Furthermore, inhaled tigecycline was effective against $M a b$ in a dose-dependent manner in granulocyte-macrophage colony-stimulating factor (GM-CSF) knockout mice (Pearce et al., 2020). The most extensive clinical trials were conducted for 52 patients with $M a b$ and M. chelonae infections to monitor the effect of tigecycline on patients. Tigecycline-containing regimens were used as a salvage treatment for patients with underlying $\mathrm{CF}$ without prior antibiotic therapy. In this study, intravenous (IV) tigecycline given ( $50 \mathrm{mg}$ daily) for $\geq 1$ month as part of a multidrug regimen resulted in a clinical remission rate of more than $60 \%$. However, adverse events were also reported in more than $90 \%$ of the cases. The general adverse effects were vomiting and nausea (Wallace et al., 2014). Furthermore, a retrospective analysis was also performed to evaluate the in vivo efficacy and adverse effects of tigecycline administration, wherein 32 of the 53 patients with Mab pulmonary disease (60\%) met the criteria for treatment success, 19 (49\%) of the 39 patients with Mab subsp. abscessus pulmonary disease met the criteria for treatment success and 13 (93\%) of the 14 patients with Mab subsp. massiliense pulmonary disease met the criteria for treatment success. However, tigecycline treatment has also resulted in severe adverse effects, mainly gastrointestinal distress, such as nausea and vomiting (in 29 of 60 cases;
48.3\%) (Chen et al., 2019). Thus, intravenous administration of tigecycline is deemed undesirable for long-term treatment of patients (Kaushik et al., 2019). Furthermore, in 2010 and 2013, the US FDA reported an increased risk of mortality associated with tigecycline use. Therefore, a new version of tigecycline with similar or better efficacy and fewer adverse effects, preferably with oral bioavailability, is desperately needed to improve the treatment for Mab infections (Kaushik et al., 2019).

For these reasons, two newly developed tigecycline analogues, omadacycline and eravacycline, have been reported to show therapeutic potential. Omadacycline has an in vitro activity against the wild-type and drug-resistant $M a b$ with an $\mathrm{MIC}_{90}$ $2 \mathrm{mg} / \mathrm{L}$ near the MIC of tigecycline. However, eravacycline showed half in vitro $\mathrm{MIC}_{90}(1 \mathrm{mg} / \mathrm{L})$ than tigecycline. Considering the steady-state area under the curve (AUC) and MICs obtained against Mab, the free drug AUC/MIC ratios for omadacycline and eravacycline, given intravenously, are expected to be approximately eight to ten times higher and twice higher than tigecycline, respectively. This improved the intravenously administered pharmacokinetic/pharmacodynamic parameters, and activity data suggest that eravacycline and omadacycline could be more effective than tigecycline clinically (Kaushik et al., 2019). Recently, oral (PO) and IV formulations of eravacycline (brand name Xerava) were clinically examined, but only the IV formulation was FDA-approved for treating complicated cIAIs in patients who are 18 years or older. Eravacycline's mechanism of action is similar to tetracycline because it blocks protein synthesis by binding to ribosomes (Lee and Burton, 2019). Omadacycline is associated with significantly less nausea and fewer treatmentemergent adverse events than tigecycline (Gotfried et al., 2017). Recently, a clinical study was reported for using omadacycline on four patients with culture-positive Mab disease (two patients had a cutaneous disease, one had the pulmonary disease and another had osteomyelitis and bacteraemia). In this report, the patients were treated with an omadacycline regimen, including other antimicrobial agents, for a median duration of 166 days. Omadacycline-containing regimens showed a clinical cure in three of the four patients. Omadacycline is relatively well tolerable during long-term treatment, although one patient discontinued therapy at the sixth month because of nausea. Despite this positive case report, further experiments are needed to determine the role of omadacycline in treating $M a b$ disease (Pearson et al., 2020).

TP-271 is a synthetic fluorocycline antibiotic belonging to tetracyclines. Like other fluorocyclines, TP-271 inhibits protein synthesis by acting on the $30 \mathrm{~S}$ ribosomal subunit. TP-271 showed in vitro activity against $M a b$ with an $\mathrm{MIC}_{90}$ range of $0.06-0.5 \mathrm{mg} / \mathrm{L}$, although this compound has not been tested for its in vivo efficacy in animal models with Mab infection. TP-271 has demonstrated broad-spectrum in vitro and in vivo activities against various community-acquired organisms, including Acinetobacter baumannii, Staphylococcus spp., Streptococcus spp., Legionella pneumophila, Haemophilus influenzae, Moraxella catarrhalis and L. pneumophila, and other biothreat pathogens (Cynamon et al., 2012). Furthermore, TP-271 also showed potent in vivo efficacy in mouse and nonhuman primate models of inhalational Francisella tularensis and Bacillus anthracis (Grossman et al., 
2017a; Grossman et al., 2017b). TP-271 is currently in phase I clinical trial, in which the safety and exposure in healthy volunteers receiving TP-271 intravenously and orally is being evaluated (Grossman et al., 2017b). Therefore, TP-271 is worth evaluating for in vivo efficacy against inhaled $M a b$ in animal models.

\section{Rifamycin}

Rifamycins were first isolated in 1957 from Streptomyces mediterranei, which was later re-named Amycolatopsis mediterranei (Sensi et al., 1959; Verma et al., 2011). Further studies showed that although rifamycin B was microbiologically inactive, its activity depended on its transformation into an active product in aqueous solutions, such as test cultures or body fluids. The transformed products, rifamycin $\mathrm{O}, \mathrm{SV}$ and $\mathrm{S}$, were then isolated and are microbiologically active. Of these, rifamycin SV had the best in vivo activity, tolerability and solubility; it has been used to treat gram-positive bacterial infections in many countries. However, rifamycin SV has poor anti-TB activity (Banerjee et al., 1992). Rifampicin targets the $\beta$-subunit of bacterial RNA polymerase (RpoB; Rv0667) in $M t b$ (Rominski et al., 2017; Ganapathy et al., 2019). In this context, rifampicin resistance is primarily caused by $r v 0667$ mutation as acquired drug resistance. Critically, rifampicin retains bactericidal activity against intra-macrophages and slow metabolising $M t b$, such as drug-tolerant and non-replicating bacteria. Furthermore, rifamycins can sterilise mycobacteria in caseum, the necrotic material at the centre of granulomas, which is difficult to eradicate. Thus, rifampicin showed strong potency against $M t b$ in vivo and reduced relapse rate, consequently shortening $\mathrm{TB}$ therapy to 6 months (Ganapathy et al., 2019) (Figure 1).

However, rifampicin is excluded from the treatment of $M a b$ lung disease owing to its low potency. It showed a high in vitro $\mathrm{MIC}_{90}$ value against all $M a b$ subspecies in cation-adjusted Mueller-Hinton broth (approximately $165 \mathrm{mg} / \mathrm{L}$ ) (Aziz et al., 2017). As rifampicin does not cause acquired resistance by $r p o B$ mutation in $M a b$, its resistance by $M a b$ is attributed to an intrinsic mechanism. Recently, Rominski et al. showed that rifampicin ADP-ribosyltransferase (MAB_0591; Arr_Mab), which catalyses ADP-ribosylation at the C23 position of rifamycin, is a significant innate rifamycin resistance in $M a b$ subsp. abscessus ATCC 19977 via gene knockout studies. Deletion of MAB_0591 improved the potency of rifamycin compared with the Mab parental type strain and the rifamycin MIC was increased when mutant was complemented with MAB_0591. Thus, Arr_Mab is the major innate rifamycin resistance determinant of Mab (Rominski et al., 2017).

Also, the Mab genome encodes many proteins, such as members of the major facilitator family, ATP-banding cassette transporters and $\mathrm{MmpL}$ proteins, which may be involved in drug efflux systems. It also encodes a small multidrug-resistant family, a family of lipophilic drug efflux proteins and a multidrugresistant stp protein involved in spectinomycin and tetracycline resistance similar to $M t b$ (Nessar et al., 2012; Sassi and Drancourt, 2014).

However, rifamycin has been recently used to manage Mab. Aziz et al. conducted in vitro screening using a collection comprising 2,662 US FDA-approved compounds and narrowed down rifabutin as a hit. This rifamycin analogue had an $\mathrm{MIC}_{90}$ of $2.5 \mathrm{mg} / \mathrm{L}$ against $M a b$ bamboo strain, three $M a b$ subspecies and clinical isolates (Aziz et al., 2017). In this context, rifabutin has been spotlighted recently. Furthermore, rifabutin showed in vivo efficacy against $M a b$ in a zebrafish model, extending the lifespan of the Mab-infected zebrafish and in NOD.CB17-Prkdcscid/NCrCrl mouse model compared with clarithromycin (Johansen et al., 2020a; Dick et al., 2020). Rifabutin treatment applications have some benefits, namely high intracellular penetration, high volume of distribution, adequate concentrations at the infection site, less drug-drug interaction and better toleration by a large proportion of patients. Furthermore, there are no antagonistic effects with other clinically used anti-Mab antibiotics that have not been reported (Ganapathy et al., 2019). In addition, the chemical structures of rifabutin are different from other rifamycin analogues. It lacks a hydroquinone moiety that $M a b$ readily metabolises at the $\mathrm{C} 1$ and $\mathrm{C} 4$ positions. Thus, this structural difference is considered a key factor for anti-Mab activity (Hanh et al., 2020b). Nonetheless, rifabutin is also a substrate of Arr, a member of rifamycin; consequently, its activity is inhibited by Arr. Thus, co-treatment with Arr-inhibitor or structural modification of rifabutin might improve its potency against Mab (Schäfle et al., 2021). Recently, another rifamycin analogue, i.e., rifamycin $\mathrm{O}$, which lacks hydroquinone in the $\mathrm{C} 1$ and $\mathrm{C} 4$ positions, also showed anti-Mab activity in an infected zebrafish model (Hanh et al., 2020b).

\section{Oxazolidinone}

Oxazolidinone is determined to have bactericidal activity against many gram-positive bacteria, such as vancomycin-intermediate strains, VRE, MRSA and penicillin-resistant pneumococci. Linezolid is the first oxazolidinone to be developed; it exhibits a high degree of in vitro activity against various gram-positive pathogens. It also inhibits bacterial growth by binding to a site on the bacterium's $50 \mathrm{~S}$ subunit $23 \mathrm{~S}$ ribosomal RNA (Figure 1). This binding prevents $70 \mathrm{~S}$ ribosomal unit formation; consequently, protein synthesis is inhibited (Rosati, 2017; Cho and Jang, 2020). Furthermore, linezolid exhibits bactericidal activity against $\mathrm{Mtb}$ and has been used to treat rifampicin-resistant and multidrugresistant TB. However, prolonged administration is often limited by long-term side effects, such as reversible myelosuppression, potentially irreversible optic and peripheral neuropathies (Cho and Jang, 2020). Linezolid has shown a weak in vitro activity against Mab infections with a modal MIC of $32 \mathrm{mg} / \mathrm{L}$ and an $\mathrm{MIC}_{90}$ of $64 \mathrm{mg} / \mathrm{L}$ (Wallace et al., 2001).

Compared with linezolid, tedizolid is a next-generation oxazolidinone with a favourable toxicity profile and superior penetration into the epithelial lining fluid. Its $\mathrm{MIC}_{50}$ and $\mathrm{MIC}_{90}$ were 1 and $4-8 \mathrm{mg} / \mathrm{L}$, respectively, across all Mabtested strains, and the values were 2-16-fold lower than those of the linezolid. The superior in vitro potency of tedizolid against $M a b$ suggests that it is a potential treatment agent for $M a b$ infections (Brown-Elliott and Wallace, 2017). Furthermore, pre-exposure of Mab complex to tedizolid sub-MICs did not initiate any drug-inducible drug resistance. Time-kill kinetics 
assays demonstrated the bacteriostatic activity of tedizolid against all Mab subspecies, even at high drug concentrations (four to eight times of the MIC) (Tang et al., 2018).

A novel oxazolidinone, delpazolid (code no. LCB01-0371) that contains cyclic amidrazone was developed by LegoChem Biosciences and showed improved safety, tolerability and pharmacokinetics (PK). It has been reported that delpazolid does not cause adverse events, such as myelosuppression, which is a severe side effect of linezolid, even after 3 weeks of repeated dosing in phase 1 clinical trial (Cho and Jang, 2020). Delpazolid has a potential broad-spectrum in vitro activity against Mab ATCC 19977 with MIC $_{50}$ of $1.2 \mathrm{mg} / \mathrm{L}$. Furthermore, it resulted in reduced bacterial load in the lungs to approximately $3.7 \log _{10}$ CFUs compare to the efficacy of linezolid at $100 \mathrm{mg} / \mathrm{kg}$. Currently, delpazolid is in phase II clinical trials for pulmonary TB. Thus, delpazolid may be a promising new class of oxazolidinones with enhanced protection that could eventually take the place of linezolid in the long-term treatment of Mab (Kim et al., 2017). Recently, Wen et al. evaluated the in vitro susceptibility of 115 isolates and 32 reference strains that are members of different RGM species against four oxazolidinones, namely, delpazolid, sutezolid, tedizolid and linezolid. The results showed that tedizolid had the most potent inhibitory activity $\left(\mathrm{MIC}_{50}=1 \mathrm{mg} / \mathrm{L}\right.$ and $\mathrm{MIC}_{90}=$ $2 \mathrm{mg} / \mathrm{L}$ ) against $\mathrm{Mab}$ in vitro. Simultaneously, delpazolid presented the best activity against Mycobacterium fortuitum, giving important insights into the potential clinical application of oxazolidinones to treat RGM infections (Wen et al., 2021). However, the use of oxazolidinone in clinical infection is not simply considered by its MIC value. Thus, PK profiles and safety issues should be compared together with in vivo infection models. However, there is no exact comparison of PK profiles, safety issues and in vivo efficacy between tedizolid and delpazolid. Delpazolid received an FDA orphan drug designation, a qualified infectious disease product designation, and was selected as a fast-track target drug (Cho and Jang, 2020).

\section{Diarylquinoline}

Bedaquiline is a diarylquinoline antibiotic that inhibits the proton pump of mycobacterial ATP synthase, resulting in ATP depletion, unstable pH homeostasis and cell death (Andries et al., 2005; Koul et al., 2007) (Figure 1). It was authorised by the FDA and the European Medicines Agency in December 2012 to treat multidrugresistant TB (MDR-TB) (Olaru et al., 2015). In vitro MIC range of bedaquiline against $M a b$ subspecies clinical isolates is $0.007-1 \mathrm{mg} /$ $\mathrm{L}$, with an $\mathrm{MIC}_{50}$ and $\mathrm{MIC}_{90}$ of 0.06 and $0.12 \mathrm{mg} / \mathrm{L}$, respectively (Brown-Elliott and Wallace, 2017). Furthermore, verapamil, a calcium channel antagonist recognised to inhibit bacterial efflux pumps, has been tested with bedaquiline to potentiate the activity of bedaquiline against Mab. In the study, Viljoen et al., reported that verapamil increased the efficacy of bedaquiline against $M a b$ clinical isolates and low-level resistant strains, both in vitro and a THP-1 macrophage infection model. Thus, the authors suggested that combining efflux pump inhibitors, such as verapamil and bedaquiline, may have clinical potential as adjunctive therapy (Viljoen et al., 2019). Bedaquiline has shown various in vivo activity against Mab-infected animal models, such as zebrafish and immunocompromised mice. Research by Dupont et al. has indicated that bedaquiline has a strong protective impact on infected zebrafish larvae from Mab-induced killing with a reduced number of abscesses and cords (Dupont et al., 2017).

Furthermore, Obregón-Henao et al., first tested the efficacy of bedaquiline using acute GKO and severe-combined immunodeficient (SCID) mouse treatment model (ObregónHenao et al., 2015). In the models, bedaquiline $(30 \mathrm{mg} / \mathrm{kg})$ significantly reduced bacterial loads in the lungs, spleens and livers 15 days after treatment. However, by contrast, another study demonstrated that bedaquiline $(25 \mathrm{mg} / \mathrm{kg})$ did not modify the decrease in bacterial burden (less than 1 $\log _{10}$ CFU) during the 2 months when its activity was evaluated in a nude mouse, that is, athymic mice with depletion of $\mathrm{T}$ cells (Lerat et al., 2014). Thus, bedaquiline efficacy in the in vivo animal model is uncertain and can depend on the animal model. However, recently Le Moigne et al., demonstrated that treatment with the bedaquiline plus imipenem combination enhanced $M a b$ clearance rather than antibiotic treatment alone in $\mathrm{C} 3 \mathrm{HeB} / \mathrm{FeJ}$ mice model. Thus, studies showed that the activity of bedaquiline can be potentiated imipenem activity in combination (Moigne et al., 2020).

In 2015, Philley et al. reported the preliminary results of bedaquiline as a salvage therapy for patients with Mab lung disease. It had a small-scale off-label use of bedaquiline for treatment failure of lung diseases caused by Mab. In the study, bedaquiline was used to treat four patients with nodular and cavity radiographic features with Mab disease. It showed clinical improvement in all cases after 3 months with bedaquiline-containing regimen treatment and without severe side effects. However, only one patient reported improved clinical symptoms after 6 months of observation (Philley et al., 2015). Thus, there is currently no clinical evidence to show that bedaquiline is a potential option for treating Mab infection. Although bedaquiline shows significant clinical activity, it might not be used for longterm Mab treatment since it is highly lipophilic, shows a long terminal half-life and has a cardiotoxicity liability associated with QT interval prolongation (Sarathy et al., 2020).

Recently, TBAJ-876, a less lipophilic bedaquiline analogue, has a higher clearance and a lower cardiotoxic potential; also, it has been evaluated for Mab. TBAJ-876 displayed submicromolar in vitro activity $\left(\mathrm{MIC}_{90}\right.$ ranged $0.2-0.7 \mathrm{mg} / \mathrm{L}$ ) against $\mathrm{Mab}$ reference strains, including three subspecies of $M a b$ and clinical isolates similar to bedaquiline. Furthermore, TBAJ-876 showed similar in vivo efficacy to bedaquiline at $10 \mathrm{mg} / \mathrm{kg}$. It reduced the bacterial burden of $M a b$ at $30 \mathrm{mg} / \mathrm{kg}$ compared with bedaquiline $(20 \mathrm{mg} / \mathrm{kg})$ in a Mab-infected mouse model (Sarathy et al., 2020). This demonstrated that TBAJ-876, with improved tolerability and $\mathrm{PK}$ profiles, may clinically aid in the treatment of Mab lung disease.

\section{Fluoroquinolones}

Fluoroquinolones are used as second-line drugs for MDR-TB, and it inhibits the supercoiling action of DNA gyrase, a unique target of fluoroquinolones (Figure 1). The fluoroquinolone antibiotics include ciprofloxacin, gemifloxacin, levofloxacin, moxifloxacin 
and ofloxacin. Fluoroquinolones are recommended for treating macrolide-resistant Mab lung disease based on drug susceptibility testing results. In vitro, ciprofloxacin and moxifloxacin have showed high activities, with 57 and $73 \%$ susceptibility for Mab isolates, respectively (Park et al., 2008). However, in vitro MIC value is high. MICs of moxifloxacin for Mab complex range from 2 to more than $8 \mathrm{mg} / \mathrm{L}\left(\mathrm{MIC}_{50}\right.$ of $8 \mathrm{mg} / \mathrm{L}$ and $\mathrm{MIC}_{90}$ of more than $8 \mathrm{mg} / \mathrm{L}$ ) (Hatakeyama et al., 2017). Although fluoroquinolone cannot be used clinically as monotherapy because of its inducible mutational resistance, both ciprofloxacin and moxifloxacin may be used as alternative oral agents for treating Mab lung disease combined with other drugs (Park et al., 2008). Since GyrB quinolone-resistance determining region (QRDR) confers resistance to fluoroquinolones in $M t b, M a b$ was also expected to comprise amino acid changes within the GyrA QRDR (Nessar et al., 2012). However, recently, Kim et al. reported that amino acid substitutions associated with fluoroquinolone resistance were unidentified in any of the Mab gyrase genes (gyrA and $B$ ) from moxifloxacin susceptible, intermediate and resistant strains (22 Mab subsp. abscessus and 24 Mab subsp. massiliense) (Kim et al., 2018). Therefore, Kim et al. explained that there is no clear correlated evidence between mutation of the DNA gyrase genes and moxifloxacin resistance in $M a b$, indicating that alternative mechanisms might be involved in moxifloxacin resistance, such as efflux pumps, which is well known in $M t b$ (Kim et al., 2018).

DC-159a is a newly synthesised broad-spectrum 8-methoxyfluoroquinolone and has shown bactericidal activities against various respiratory pathogens, including multidrug-resistant Streptococcus pneumoniae and quinolone-resistant strains (Clark et al., 2008; Hoshino et al., 2008). Furthermore, DC-159a showed potent in vitro activity against quinolone-resistant multidrug-resistant $M t b\left(\mathrm{MIC}_{90}, 0.5 \mathrm{mg} / \mathrm{L}\right)$ and drug-susceptible isolates $\left(\mathrm{MIC}_{90}, 0.06 \mathrm{mg} / \mathrm{L}\right)$. In the in vitro activity comparison with $M t b$, DC-159a was 4-32-fold more potent than other quinolones (Disratthakit and Doi, 2010). DC-159a also showed the highest activities against NTM, such as M. fortuitum, MAC, M. chelonae and M. abscessus. The MIC range of DC-159a against $M a b$ was $4-32 \mathrm{mg} / \mathrm{L}$, which is $2-4$-fold lower than those of other tested quinolones, such as moxifloxacin, levofloxacin and gatifloxacin (Disratthakit and Doi, 2010). However, its activity is unsatisfactory compared with other anti-Mab candidates because the in vitro MIC value is higher than others. Still, there is no in vivo efficacy data for Mab (Soni et al., 2016).

\section{Clofazimine}

Clofazimine (CFZ) belongs to the riminophenazine group and has demonstrated an impressive activity against rapid-growing Mycobacterium (Mab, M. fortuitum and M. smegmatis) and slowgrowing Mycobacterium (Mtb, MAC and M. leprae) (Cholo et al., 2017). Most Mab clinical isolates had CFZ MICs of $\leq 1 \mathrm{mg} / \mathrm{L}$ (Wu et al., 2018). Recently, CFZ has been used for Mab treatment (Martiniano et al., 2017; Yang et al., 2017). Furthermore, CFZ has shown a synergistic effect with other antimicrobial agents, including amikacin and clarithromycin, against $M$. avium and $M a b$ in vitro. CFZ has several advantages as an effective drug because it has a long half-life, slow metabolic elimination, low cost, high concentration in macrophages and rapid localisation within phagocytes (Shen et al., 2010). However, CFZ has no exact mechanism of action (MOA), and information on its resistance in patients is yet undetermined (Figure 1). Although the exact MOA for CFZ is not fully understood, the cell membrane seems to be the primary action site. It has been demonstrated that CFZ is a prodrug and can disrupt bacterial membrane via interaction with intracellular redox cycling, releasing reactive oxygen species (Yano et al., 2011). Furthermore, another putative MOA is that CFZ stimulates phospholipase A2 (PLA2) activity, resulting in an accumulation of detergent-like lysophospholipids and disrupting fundamental cellular functions. Also, it has been hypothesised that it inhibits cell replication by binding to the guanine base of deoxyribonucleic acid (McGuffin et al., 2017). However, no study has been conducted to examine $\mathrm{CFZ}$ resistance in patients, and the MOA of CFZ in Mab remains unestablished. Recently, Yuanyuan et al. investigated the resistance mechanism of CFZ using 29 laboratory-induced CFZ-resistant Mab strains through whole-genome sequencing, wherein three genes (MAB_2299c, MAB_1483 and MAB_0540) were identified to be most commonly associated with CFZ resistance. However, future studies are needed to address the role of the identified mutations (Chen et al., 2018). CFZ shares a resistant mechanism with BDQ by up-regulating MmpL5 due to mutations in Rv0678, which is the transcriptional regulator in $M t b$. Besides, loss-of-function mutations in pepQ (Rv2535c) that encode putative Xaa-Pro aminopeptidase show four times higher MICs than those for the H37Rv control in the MIC determination test with CFZ and BDQ. However, the exact mechanism by which mutations in pepQ provide BDQ and CFZ cross-resistance remains unclear (Andries et al., 2014; Almeida et al., 2016). Based on the BLAST similarity match, Rv0678 and PepQ (Rv2535c) have 33 and $66 \%$ amino acid sequence identity with MAB_1857c and MAB_2838c, respectively.

There are some positive in vivo results with CFZ for the Mabinfected animal model. Drosophila infected with Mab had its lifespan extended by 3 days after treatment with CFZ (Oh et al., 2014). Furthermore, Andrés et al. evaluated diverse mice infection models, such as nude (nu/nu), SCID, GKO and GMCSF knockout mice. When GKO mice were used for Mab in vivo study, CFZ treatment significantly reduced bacterial burden in the lung, spleen and liver after five and 15 days of treatment. These anti-mycobacterial activities were enforced when CFZ was combined with bedaquiline (Obregón-Henao et al., 2015). Also, there are some retrospective studies of CFZ treatment for Mabinfected patients. Yang et al. reported a retrospective study involving 42 patients with Mab lung disease treated with CFZcontaining regimens between November 2013 and January 2015. In the study, CFZ achieved $24 \%$ sputum-negative culture conversion after CFZ-containing antibiotic treatment, including symptoms and radiographic improvement. Thus, the authors suggested that CFZ-containing regimens improve treatment outcomes for patients with Mab lung disease (Yang et al., 2017). Besides, Carey et al. also performed a retrospective cohort analysis of patients infected with Mab subsp. abscessus/ bolletii/massiliense, M. fortuitum or M. chelonae. The patients 
were treated with a CFZ-containing regimen for over 7 years in an institution. During each treatment course, CFZ was given along with a median of five other antibiotics. Interestingly, treatment with the initial regimen was achieved in $43 \%$ of patients with pulmonary infection and $71 \%$ of patients with a nonpulmonary infection. As a component of multidrug therapy for both pulmonary and nonpulmonary RGM infections, CFZ was safe and relatively well tolerated (Carey et al., 2019).

\section{Inhalation Route: Nitric Oxide/Liposomal Amikacin/Molgramostim}

Nitric oxide (NO) is naturally synthesised by a NO synthase in mammalian cells. No synthase activity is upregulated in the pulmonary system after infection or stimulation by cytokines in normal subjects (Xue et al., 2018). In CF patients, the increase of airway NO level results in improvement of lung function (Grasemann et al., 1997; Ho et al., 1998; Keen et al., 2010). Furthermore, NO has been shown to act as a broad-range antimicrobial agent, seen in vitro, ex vivo and animal infection models (Goldbart et al., 2020). Yaacoby-Bianu et al., previously reported that adjunctive-inhaled nitric oxide (iNO) therapy at $160 \mathrm{ppm}$ for $30 \mathrm{~min}$, five times/day for up to 26 days to CF patients resulted in reduced $M a b$ load in sputum and improvement in pulmonary function (Yaacoby-Bianu et al., 2018). Furthermore, iNO treatment increased time to positivity in Mycobacterium culture with safety and tolerability albeit Mab culture conversion was not achieved (Bentur et al., 2020). As shown in Table 1, the iNO for patients with Mab has recently completed phase II clinical trials and pilot study to assess the effect of intermittent iNO on the treatment of NTM lung infection in $\mathrm{CF}$ and non-CF patients are currently recruiting in its clinical phase (Wu et al., 2018).

Amikacin is well known as one of the most active antibiotics for treating $M a b$ pulmonary disease through the combination of one or more parenteral drugs (cefoxitin, imipenem or tigecycline) (Lee et al., 2017). Furthermore, it shows concentration-dependent bactericidal effects through irreversibly binding to the bacterial $30 \mathrm{~S}$ ribosomal subunit, specifically in contact with $16 \mathrm{~S}$ rRNA and S12 protein (Shi et al., 2013) (Figure 1). However, amikacin accumulates poorly in cells, which its efficacy is limited against intracellular infections (Zhang et al., 2018). The method for the targeted delivery of amikacin in the host cell is to package the antibiotic into liposomes, sphere-shaped vesicles with a membrane composed of a phospholipid bilayer (Akbarzadeh et al., 2013). The liposomes are used for drug delivery due to their unique properties, such as small size, low toxicity, tissue/cell targeting (Beltrán-Gracia et al., 2019). Based on these advantages, liposome encapsulation has been applied to amikacin to improve its killing ability in treating intracellular $M a b$ infections in macrophages. When macrophages infected with the Mab were treated with amikacin and liposomal amikacin for inhalation (LAI), LAI at $10 \mathrm{mg} / \mathrm{L}$ showed significant effectiveness than free amikacin for intracellular Mab (Rose et al., 2014). This intracellular activity was further assessed in patients with treatment-refractory pulmonary NTM disease. In a clinical phase II study for Mab-infected patients, of four patients who achieved sputum culture conversion, three were converted after receiving LAI and one while receiving placebo at 28 days end-of-study follow-up visit. Furthermore, among the four patients who were converted, two had negative cultures 12 months after LAI discontinuation, one reverted to positive cultures, and one did not consent to participate in the 12-month follow-up phase (Olivier et al., 2017). Its efficacy and safety profiles are being further evaluated in a phase II trial against Mab. Currently, clinical phase II trials, especially LAI efficacy in $M a b$ patients, are recruiting (Table 1).

Inhaled molgramostim is a form of GM-CSF. GM-CSF is a protein that occurs naturally in the human immune system and plays a crucial role in activating the immune system to kill bacteria. The altered immune system by molgramostim may prevent the build-up of harmful bacteria, such as NTM in the lungs (Degiacomi et al., 2019). A phase II clinical trial was conducted to test the effectiveness of inhaled mogramostim against NTM, including Mab, in adults with CF (Table 1). However, no $M a b$-infected patient achieved sputum culture conversion after 48 -week treatment. Inversely, the participants experienced serious side effects during the treatment, and the most common adverse effect was the aggravation of bronchiectasis. The full result of inhaled molgramostim from the clinical phase II trial will be released soon (Silva, 2020).

\section{Gallium}

Iron is essential for bacteria to mediate many key processes, such as DNA synthesis, general metabolism, electron transport and oxidative stress resistance (Andrews et al., 2003). Gallium (Ga) has a nearly identical ionic radius as iron, and some bacterial uptake systems are unable to distinguish gallium from iron. Iron is uptaken by bacterial cells, and it undergoes reduction for participation in redox cycling, which is essential for life. However, Ga cannot be reduced under normal physiological conditions within the cell, therefore, disrupting iron-dependent processes (Crunkhorn, 2018; Goss et al., 2018). For this reason, many studies have demonstrated that gallium compounds can be used as antibacterial agents against many human pathogens, including multidrug-resistant CF clinical isolates (Goss et al., 2018). $\mathrm{Ga}$ in the form of $\mathrm{Ga}\left(\mathrm{NO}_{3}\right)_{3}$ is an FDA-approved drug for treating hypercalcaemia of malignancy (Warrell et al., 1986). Recently, Abdalla et al. assessed the growth inhibitory activity of different types of $\mathrm{Ga}$ compound against wild-type $M a b$ and clinical isolates obtained from $\mathrm{CF}$ and other patients. They demonstrated that Ga-protoporphyrin is the most potent type of Ga compound in vitro and intracellularly, and it completely inhibited the Mabs at much lower concentrations than $\mathrm{Ga}\left(\mathrm{NO}_{3}\right)_{3}$ (Abdalla et al., 2015). Furthermore, nanoparticles encapsulating $\mathrm{Ga}$ meso-tetraphenylporphyrine (GaTP) showed greater bactericidal activity than $\mathrm{Ga}\left(\mathrm{NO}_{3}\right)_{3}$ against $M a b$ in vitro and intracellularly (Choi et al., 2018). Also, they evaluated the effect of drug and drug combination with $\mathrm{Ga}\left(\mathrm{NO}_{3}\right)_{3}$ and $\mathrm{Ga}$ porphyrin $(\mathrm{GaPP})$ against $\mathrm{Mab}$ in vitro and in a murine pulmonary model with $M a b$ infection. In this study, the authors observed that $\mathrm{Ga}\left(\mathrm{NO}_{3}\right)_{3}$ combined with $\mathrm{GaPP}$ showed significantly potent synergistic inhibitory activity against $M a b$. These findings suggest that combinations of different $\mathrm{Ga}$ compounds can be 
synergistically used for anti-Mab treatment in the clinic (Choi et al., 2020). Currently, Ga evaluates its safety and tolerability in adult patients with CF infected with NTM, including $M a b$ as phase I (Table 1).

\section{MmpL3 Inhibitors}

MmpL3 is highly conserved across the mycobacteria. It is responsible for translocating mycolic acids in a trehalose monomycolate (TMM) form across the inner membrane, thus playing an essential role in cell wall synthesis (Figure 1). TMM molecules are precursors of trehalose dimycolate (TDM; cord factor) or to arabinogalactan yielding wall-bound mycolates (McNeil et al., 2020; Sarathy et al., 2020). The mmpL3 knockdown strain of $M t b$ led to a failure of cell division and, consequently rapid bacterial death due to TMM accumulation (Degiacomi et al., 2017). Recently, several MmpL3 inhibitors have been identified from the phenotypic screening of compound libraries against $M a b$ and MAC. The availability of bactericidal inhibitors that target MmpL3 in Mab will provide opportunities to treat pulmonary Mab infections (Li et al., 2018).

\section{PIPD1}

PIPD1 is defined as a new piperidinol-based molecule. It targets the mycolic acid transporter, MmpL3, which is required to transport TMMs and abrogate the mycolylation of arabinogalactan (Dupont et al., 2016). PIPD1 shows a potential broad-spectrum in vitro activity against smooth (S) and rough (R) $M a b$ CIP104536 with a MIC of $0.125 \mathrm{mg} / \mathrm{L}$. The range of $\mathrm{MBC}_{99}$ (the concentration of the drug at which $99 \%$ of input bacilli were killed) against $M a b \mathrm{~S}$ is $0.125-0.5 \mathrm{mg} / \mathrm{L}$. In the $M a b$-zebrafish model, the treatment of infected zebrafish with PIPD1 showed increased embryo survival and decreased bacterial burden. Besides, MAB_4508 encoding a protein homologous to MmpL3 is detected as a resistant mutation to PIPD1 (Dupont et al., 2016).

\section{Indole-2-Carboxamides}

Indole-2-carboxamides (ICs) are another MmpL3 inhibitor and have activity against a broad spectrum of NTM pathogens. Two IC derivatives (compounds 5 and 25) prevented mycolic acid translocation from the cytoplasm to the periplasmic space by inhibiting MmpL3, consequently inducing bacterial death. Both compounds showed excellent in vitro activity against wild-type Mab ATCC 19977 (MIC of compound $5=0.25 \mathrm{mg} / \mathrm{L}$ and compound $25=0.063 \mathrm{mg} / \mathrm{L}$ ) (Franz et al., 2017). Compounds 25- and 5-resistant mutant harbours a missense mutation (A309P) in the MmpL3 protein and this mutant showed 16-128fold increase in MICs of compounds 25 and 5, respectively (MIC of compound 5 against the A309P missense mutant $=32 \mathrm{mg} / \mathrm{L}$; MIC of compound 25 against the A309P mutant $=1 \mathrm{mg} / \mathrm{L}$ ) (Pandya et al., 2019). Both derivatives showed statistically significant reduced bacterial load in the lung and spleen of Mab-infected mice in the acute SCID treatment mouse model. This in vivo effectiveness of ICs compounds was similar to amikacin used as a positive control (Pandya et al., 2019). The sequencing result of the MmpL3 gene from laboratory-induced resistant mutants against high-dose PIPD1 or on indole-2-carboxamides revealed a common Ala309Pro substitution in MmpL3. Additionally, the overexpression of
MmpL3 carrying the Ala309Pro mutation in Mab showed highlevel resistance to PIPD1 and indole-2-carboxamides (Dupont et al., 2016; Kozikowski et al., 2017).

In other studies with IC analogues by Kozikowski et al., lead compounds 6 and 12, which have favourable absorption, distribution, metabolism and excretion properties, also exhibited strongly in vitro activity against $M a b$ clinical isolates from $\mathrm{CF}$ and non-CF patients and especially compound 12 showed significantly reduced number of $M a b$-infected cells. The biochemical assay revealed that treatment of compound 12 strongly accumulates TMM on thin-layer chromatography, resulting in the defect of trehalose dimycolate production (TDM) synthesis, consequently failure of mycolylation of arabinogalactan (Kozikowski et al., 2017). These compounds 6 and 12 were further studied in combinations with imipenem and cefoxitin in vitro by checkerboard assay to evaluate their synergistic effect against Mab. Raynaud et al., demonstrated that combination between IC (compounds 6 and 12) plays a synergistic role with imipenem and cefoxitin in vitro and, especially compound 12 also showed synergistic effect with imipenem in THP-1 macrophages. This potential synergistic effect requires further pre-clinical animal study (Raynaud et al., 2020b).

\section{EJMCh-6}

EJMCh-6, which is a benzimidazole analogue targeting MmpL3 in $M a b$, shows a potential broad-spectrum in vitro activity against smooth (S) Mab CIP $104536^{\mathrm{T}}$ with a MIC of $0.125 \mathrm{mg} / \mathrm{L}$. EJMCh-6 is bacteriostatic in vitro and this compound showed potent activity with MIC values ranging from 0.031 to $1 \mathrm{mg} / \mathrm{L}$ against the various strains and subspecies of $M a b$ that were isolated from patients with or without CF. In the THP-1 cell model of infection with Mab, EJMCh- 6 exerted a very strong activity against intramacrophage-residing $M a b$. In the $M a b$ zebrafish model, approximately $80 \%$ of the treated embryos survive at $12 \mathrm{dpi}$ after being treated with $0.75 \mathrm{mg} / \mathrm{L}$ EJMCh-6 (Raynaud et al., 2020a).

\section{Benzothiazole Amide Compounds}

Benzothiazole Amide Compounds (CRS400226, CRS400153, CRS400359 and CRS400393) target MmpL3 and further showed excellent in vitro activity against $M a b$ infections with an $\mathrm{MIC}_{90}$ of $0.5 \mathrm{mg} / \mathrm{L}$. Among those analogues, Mary et al. assessed the in vivo efficacy of CRS400226 against Mab in chronic lung infection using GM-CSF mice for 28 days. Intratracheal administration of CRS400226 at $25 \mathrm{mg} / \mathrm{kg} /$ day for 28 days resulted in $0.64 \log _{10}$ CFU reduction compared with the vehicle control. The histological assay revealed that CRS400226-treated animals had only minor areas of airwaycentric inflammation (De Groote et al., 2018).

\section{Newly Discovered Anti-Mab Inhibitors Leucyl-tRNA Synthetase Inhibitor (Epetraborole and Benzoxaborole EC/11770)}

Epetraborole (also known as AN 3365; GSK 2251052) is a benzoxaborole analogue and leucyl-tRNA synthetase inhibitor (O’Dwyer et al., 2015; Purnapatre et al., 2018). It has shown a novel mode of action against gram-negative bacterial infections, 
such as urinary tract infections. In previous studies, the benzoxaborole analogue showed antitubercular activity against $M t b$ and M. smegmatis (Palencia et al., 2016). Recently, Kim et al. performed in vitro dual-screen against $M a b \mathrm{R}$ and $\mathrm{S}$ variants using a pandemic response box that comprises 400 structurally diverse compounds (201 antibacterials, 153 antivirals and 46 antifungals) and is a drug library assembled by the Medicines for Malaria Venture (MMV) (Kim et al., 2021). Through the screen, the authors identified epetraborole, which showed significant activity against $M a b$ wild-type strain growth in three subspecies, drug-resistant strains, clinical isolates in vitro ( $\mathrm{MIC}_{90}$ ranges from 0.1 to $1.35 \mathrm{mg} / \mathrm{L}$ ), intracellular and in the zebrafish infection model. However, according to ClinicalTrials.gov in 2017, a clinical phase II study of epetraborole for treating complicated urinary tract infection and intra-abdominal infection was terminated due to the rapid emergence of drug resistance during treatment (Purnapatre et al., 2018). Recently, another benzoxaborole analogue named benzoxaborole EC/11770 was also identified against $M a b$ by testing advanced compounds that already showed activity against $M t b$. The EC/11770 showed antibacterial activity against $M a b$ and M. avium complexes. Furthermore, EC/11770 inhibited the growth of the Mab biofilm in vitro model and showed effective in vivo efficacy in the NOD SCID mice lung infection model. Fortunately, EC/11770 showed low-resistant mutant frequency, and leucyl-tRNA synthetase was confirmed as its target (Ganapathy et al., 2021).

\section{AR-12 (OSU-03012)}

AR-12 (OSU-03012) is a novel derivative of celecoxib that inhibits phosphoinositide-dependent kinase-1 (PDK1) activity in different cell models and has further progressed to phase I clinical trial as an anticancer agent. Intriguingly, it exhibits antipathogenic activity against bacteria, fungi and viruses. According to mechanistic studies, AR-12 represses the host cell chaperone machinery, thus preventing proper viral protein folding and effective viral assembly. AR-12 also causes autophagy, which aids in clearing intracellular viruses, unfolded proteins or both (Abdulrahman et al., 2017). In the aspect of Mab activity, AR-12 displayed broad anti-Mab activity against 194 clinical Mab isolates (148 subsp. abscessus and 46 subsp. massiliense) with a moderate MIC value ( $\mathrm{MIC}_{90}$ of 4 and $8 \mathrm{mg} / \mathrm{L}$ ). Furthermore, AR-12 showed growth inhibitory activity against $M a b$ residing within primary peritoneal macrophages. Lastly, AR-12 inhibited Mab replication in a mouse model with a lung infection. AR-12 $(50 \mathrm{mg} / \mathrm{kg})$ caused significant reductions of approximately $3.7 \log _{10} \mathrm{CFU}$ in the lung after 2 weeks of treatment. The histological assay also revealed a reduction in inflammatory pathology and bacterial counts in the lungs of AR-12-treated mice compared with untreated mice (Zhang et al., 2020).

\section{SPR719}

SPR719 (previously VXc-486) is a novel aminobenzimidazole that targets ATPase subunits (GyrB/ParE) in Mtb (Locher et al., 2015). It is a novel class that targets the ATPase subunits of gyrase by a mechanism distinct from fluoroquinolones (Verma et al.,
2020). The $\mathrm{MIC}_{90}$ value for subspecies Mab subsp. abscessus, $M$ $a b$ subsp. massiliense and M. abscessus/massiliense hybrid was 2-4 mg/L. Although Mab contains a natural A92S mutation in its gyrB, the potency of SPR719 against Mab in the MIC test was greater than that of moxifloxacin, which was used as a control (Locher et al., 2015). Recently, Rubio et al. (2018) reported the in vivo efficacy result of SPR720 (a prodrug of SPR719) using a SCID mice model of infection with Mab subsp. bolletii (strain 103) at ASM Microbe 2018. Treatment was administered via oral dosing $(25,50,100,200,300$ and $400 \mathrm{mg} / \mathrm{kg} /$ day $)$. After 16 days of treatment, the bacterial burden in the lung, spleen and liver was enumerated. From the study, a daily dosage of $100 \mathrm{mg} / \mathrm{kg}$ demonstrated the most significant bacterial reduction in the lung, spleen and liver compared with that in the control group (Rubio A, Stapleton M, Verman D).

\section{Etamycin}

Etamycin (also called viridogrisein) is a cyclic peptide antibiotic isolate of marine actinomycete. Recently, etamycin showed potent activity against wild-type Mab, three subspecies of the Mab complex and clinical isolates, including the $\mathrm{R}$ and $\mathrm{S}$ variant, at an $\mathrm{MIC}_{50}$ level of $1.6-7.2 \mathrm{mg} / \mathrm{L}$. Furthermore, etamycin inhibited the growth of $M a b$ that resides in macrophages without cytotoxicity. The in vivo efficacy of etamycin in the zebrafish infection model was greater than that of clarithromycin. ZF's survival rate of $44 \mathrm{mg} / \mathrm{L}(50 \mu \mathrm{M})$ etamycin treatment was $85 \%$, higher than that observed for the treatment with $37.4 \mathrm{mg} / \mathrm{L}(50 \mu \mathrm{M})$ clarithromycin at 13 days post-infection (Hanh et al., 2020a).

\section{Thiostrepton}

Thiostrepton is an FDA-approved antimicrobial drug for animal use and is a quinaldic acid moiety containing a natural thiopeptide. It has been remarkably determined to be an effective translational blocker that binds to nucleotides A1065 and A1095 on helices 43 and 44 of the $23 \mathrm{~S}$ rRNA. Also, it binds proline residues within the $\mathrm{N}$-terminal domain of $\mathrm{uL11}$ that is a ribosomal protein. Thiostrepton has antibacterial activity against Staphylococcus aureus (MRSA), methicillin-resistant Enterococcus faecium, penicillin-resistant Streptococcus pneumoniae, vancomycinresistant enterococci, $M t b$ and $M$. marinum. Thiostrepton significantly inhibited the growth of $M a b$ in vitro and macrophage-infected Mab. Furthermore, thiostrepton significantly decreased proinflammatory cytokine production in macrophages, suggesting an inhibitory effect of thiostrepton on inflammation-induced during Mab infection. Also, thiostrepton exhibits antimicrobial effects in vivo in zebrafish models of Mab infection (Kim et al., 2019). Nevertheless, thiostrepton has limitations for its clinical use. It has a large molecular size, lacking bioavailability and poor aqueous solubility (Just-Baringo et al., 2014). Recently, newly developed semisynthetic thiopeptide, LFF571, by Novartis showed an improved pharmacokinetic profile, such as aqueous solubility, compared to thiostrepton. LFF571 tested for Clostridium difficile, which causes intestinal infections in humans. LFF571 showed excellent efficacy in a hamster model with a lower dose and fewer recurrences than vancomycin (Trzasko et al., 2012). 


\section{CONCLUSION}

$M a b$ is described as an environmentally derived opportunistic pathogen that likely causes lung and skin infections in patients with a weak immune system in various environments. Mab infection can be acquired when taking a shower from the showerhead and during cosmetic surgery and acupuncture. Therefore, it is currently essential to proactively identify the potential severity of Mab infection in communities and healthcare institutions. It is also important to re-evaluate the case of Mab misdiagnosed as TB to determine the current infection situation of $M a b$ accurately. Presently, the most crucial aspect of treating $M a b$ is the formation of a sputum smear transition quickly. As mentioned above, various antibiotics, including anti-TB drugs, are currently used to treat $M a b$ through combination therapy, but only a few drugs can derive sputum smear conversion. Therefore, developing new drug recombination using various antibiotics, including newly identified anti-TB drugs and various efflux pump inhibitors, may be a priority.

Next, the development of new antibiotics using new libraries would be essential. Lipinski's rule-of-five (Ro5: not more than five and one hydrogen bond donors and hydrogen bond acceptors, respectively, and a partition coefficient $(\log \mathrm{P})$ value less than 5$)$ has been used for selecting candidates with good oral bioavailability properties. However, the Ro5 sometimes cannot explain the bioactivity of natural products that systematically break the Ro5 with bioavailability and bioactivity. Thus, screening natural products with expanded chemical diversity for discovering new drugs would be an alternative step for drug development against $M a b$. Natural compounds with bioavailability and bioactivity that can penetrate membranes of host cells and kill intracellular Mab would be new weapons that will expand the success rate in a drug screen. Drug screen model systems for excavating these substances are also very crucial. Developing new drugs for Mab through a new screen model that mimic the human environment is needed.

Investing in pharmaceutical companies for $M a b$ is currently passive. This is probably because there are fewer economic benefits for chronic diseases, such as cancer, diabetes, and degenerative neurological and brain diseases. Therefore, research in small- and medium-sized enterprises and

\section{REFERENCES}

Abate, G., Hamzabegovic, F., Eickhoff, C. S., and Hoft, D. F. (2019). BCG Vaccination Induces M. Avium and M. Abscessus Cross-Protective Immunity. Front. Immunol. 10, 234. doi:10.3389/fimmu.2019.00234

Abdalla, M. Y., Switzer, B. L., Goss, C. H., Aitken, M. L., Singh, P. K., and Britigan, B. E. (2015). Gallium Compounds Exhibit Potential as New Therapeutic Agents against Mycobacterium Abscessus. Antimicrob. Agents Chemother. 59, 4826-4834. doi:10.1128/AAC.00331-15

Abdulrahman, B. A., Abdelaziz, D., Thapa, S., Lu, L., Jain, S., Gilch, S., et al. (2017). The Celecoxib Derivatives AR-12 and AR-14 Induce Autophagy and clear Prion-Infected Cells from Prions. Sci. Rep. 7, 17565. doi:10.1038/s41598-01717770-8

Adjemian, J., Olivier, K. N., and Prevots, D. R. (2018). Epidemiology of Pulmonary Nontuberculous Mycobacterial Sputum Positivity in Patients with Cystic Fibrosis in the United States, 2010-2014. Ann. ATS 15, 817-826. doi:10.1513/AnnalsATS.201709-727OC universities is currently leading to a small number of studies on $M a b$ infections and antibiotics. However, a report by the United Kingdom government says the number of deaths from antibiotic-resistant bacteria by 2050 will far outweigh the number of deaths caused by chronic diseases, such as cancer, diabetes and diarrhoeal disease. Without antibiotics, we cannot perform surgery on premature infants, treat diseases like cancer and perform organ transplants and plastic surgery. Lung disease caused by Mab infection is steadily increasing worldwide; and due to an increase in the population of elderly individuals through an ageing society, and the increase in long-term care patients, $M a b$ infection is seen to be a global public health concern. Therefore, developing new drugs for $M a b$, which minimises side effects, is a challenge that must be undertaken. Significant investment must be made to develop new drugs and fundamental research on $M a b$, which is resistant to various antibiotics. Thus, this will lead to the screening of Mabeffective drugs in clinical trials.

\section{AUTHOR CONTRIBUTIONS}

JJ conceptualised the research work. NQ and JJ searched and gathered the previous studies. JJ wrote the manuscript. NQ and JJ critically reviewed the manuscript. NQ and JJ edited the reviewed manuscript. JJ critically evaluated and revised the manuscript and supervised the whole project.

\section{FUNDING}

This research was supported by the National Research Foundation of Korea (grants 2020R1A2C1004077).

\section{ACKNOWLEDGMENTS}

Authors would like to thank all scientists who are developing novel anti-Mab agents.

Akbarzadeh, A., Rezaei-Sadabady, R., Davaran, S., Joo, S. W., Zarghami, N., Hanifehpour, Y., et al. (2013). Liposome: Classification, Preparation, and Applications. Nanoscale Res. Lett. 8, 102. doi:10.1186/1556-276X8-102

Almeida, D., Ioerger, T., Tyagi, S., Li, S.-Y., Mdluli, K., Andries, K., et al. (2016). Mutations in pepQ Confer Low-Level Resistance to Bedaquiline and Clofazimine in Mycobacterium tuberculosis. Antimicrob. Agents Chemother. 60, 4590-4599. doi:10.1128/AAC.00753-16

Andrew, E. C., Connell, T., Robinson, P., Curtis, N., Massie, J., Robertson, C., et al. (2019). Pulmonary Mycobacterium Abscessus Complex in Children with Cystic Fibrosis: A Practical Management Guideline. J. Paediatr. Child. Health 55, 502-511. doi:10.1111/jpc.14427

Andrews, S. C., Robinson, A. K., and Rodríguez-Quiñones, F. (2003). Bacterial Iron Homeostasis. FEMS Microbiol. Rev. 27, 215-237. doi:10.1016/S0168-6445(03) 00055-X

Andries, K., Verhasselt, P., Guillemont, J., Göhlmann, H. W. H., Neefs, J. M., Winkler, H., et al. (2005). A Diarylquinoline Drug Active on the ATP Synthase of Mycobacterium tuberculosis. Science 307, 223-227. doi:10.1126/science.1106753 
Andries, K., Villellas, C., Coeck, N., Thys, K., Gevers, T., Vranckx, L., et al. (2014). Acquired Resistance of Mycobacterium tuberculosis to Bedaquiline. PLoS One 9, e102135. doi:10.1371/journal.pone.0102135

Aziz, D. B., Low, J. L., Wu, M.-L., Gengenbacher, M., Teo, J. W. P., Dartois, V., et al. (2017). Rifabutin Is Active against mycobacterium Abscessus Complex. Antimicrob. Agents Chemother. 61, e00155-17. doi:10.1128/AAC.00155-17

Banerjee, U. C., Saxena, B., and Chisti, Y. (1992). Biotransformations of Rifamycins: Process Possibilities. Biotechnol. Adv. 10, 577-595. doi:10.1016/ 0734-9750(92)91454-M

Beltrán-Gracia, E., López-Camacho, A., Higuera-Ciapara, I., Velázquez-Fernández, J. B., and Vallejo-Cardona, A. A. (2019). Nanomedicine Review: Clinical Developments in Liposomal Applications. Cancer Nano 10. doi:10.1186/ s12645-019-0055-y

Bentur, L., Gur, M., Ashkenazi, M., Livnat-Levanon, G., Mizrahi, M., Tal, A., et al. (2020). Pilot Study to Test Inhaled Nitric Oxide in Cystic Fibrosis Patients with Refractory Mycobacterium Abscessus Lung Infection. J. Cystic Fibrosis 19, 225-231. doi:10.1016/j.jcf.2019.05.002

Bernut, A., Le Moigne, V., Lesne, T., Lutfalla, G., Herrmann, J.-L., and Kremer, L. (2014). In VivoAssessment of Drug Efficacy against Mycobacterium Abscessus Using the Embryonic Zebrafish Test System. Antimicrob. Agents Chemother. 58, 4054-4063. doi:10.1128/AAC.00142-14

Blauwendraat, C., Dixon, G. L. J., Hartley, J. C., Foweraker, J., and Harris, K. A. (2012). The Use of a Two-Gene Sequencing Approach to Accurately Distinguish between the Species within the Mycobacterium Abscessus Complex and Mycobacterium chelonae. Eur. J. Clin. Microbiol. Infect. Dis. 31, 1847-1853. doi:10.1007/s10096-011-1510-9

Brown-Elliott, B. A., Vasireddy, S., Vasireddy, R., Iakhiaeva, E., Howard, S. T., Nash, K., et al. (2015). Utility of Sequencing Theerm(41) Gene in Isolates of Mycobacterium Abscessus Subsp. Abscessus with Low and Intermediate Clarithromycin MICs. J. Clin. Microbiol. 53, 1211-1215. doi:10.1128/JCM.02950-14

Brown-Elliott, B. A., and Wallace, R. J. (2017). In Vitro susceptibility Testing of Tedizolid against Nontuberculous Mycobacteria. J. Clin. Microbiol. 55, 1747-1754. doi:10.1128/JCM.00274-17

Bryant, J. M., Grogono, D. M., Greaves, D., Foweraker, J., Roddick, I., Inns, T., et al. (2013). Whole-genome Sequencing to Identify Transmission of Mycobacterium Abscessus between Patients with Cystic Fibrosis: A Retrospective Cohort Study. The Lancet 381, 1551-1560. doi:10.1016/ S0140-6736(13)60632-7

Carey, G. B., Tebas, P., Vinnard, C., Kim, D., Hadjiliadis, D., Hansen-Flaschen, J., et al. (2019). Clinical Outcomes of Clofazimine Use for Rapidly Growing Mycobacterial Infections. Open Forum Infect. Dis. 6, ofz456. doi:10.1093/ofid/ofz456

Chen, J., Zhao, L., Mao, Y., Ye, M., Guo, Q., Zhang, Y., et al. (2019). Clinical Efficacy and Adverse Effects of Antibiotics Used to Treat mycobacterium Abscessus Pulmonary Disease. Front. Microbiol. 10, 1977. doi:10.3389/ fmicb.2019.01977

Chen, Y., Chen, J., Zhang, S., Shi, W., Zhang, W., Zhu, M., et al. (2018). Novel Mutations Associated with Clofazimine Resistance in mycobacterium Abscessus. Antimicrob. Agents Chemother. 62, e00544-18. doi:10.1128/ AAC.00544-18

Cho, Y. L., and Jang, J. (2020). Development of Delpazolid for the Treatment of Tuberculosis. Appl. Sci. 10, 2211. doi:10.3390/app10072211

Choi, S.-r., Britigan, B. E., Switzer, B., Hoke, T., Moran, D., and Narayanasamy, P. (2018). In Vitro Efficacy of Free and Nanoparticle Formulations of Gallium(III) Meso-Tetraphenylporphyrine against Mycobacterium avium and Mycobacterium Abscessus and Gallium Biodistribution in Mice. Mol. Pharmaceutics 15, 1215-1225. doi:10.1021/acs.molpharmaceut.7b01036

Choi, S.-r., Switzer, B., Britigan, B. E., and Narayanasamy, P. (2020). Gallium Porphyrin and Gallium Nitrate Synergistically Inhibit Mycobacterial Species by Targeting Different Aspects of Iron/Heme Metabolism. ACS Infect. Dis. 6, 2582-2591. doi:10.1021/acsinfecdis.0c00113

Cholo, M. C., Mothiba, M. T., Fourie, B., and Anderson, R. (2017). Mechanisms of Action and Therapeutic Efficacies of the Lipophilic Antimycobacterial Agents Clofazimine and Bedaquiline. J. Antimicrob. Chemother. 72, 338-353. doi:10.1093/jac/dkw426

Chopra, S., Matsuyama, K., Hutson, C., and Madrid, P. (2011). Identification of Antimicrobial Activity Among FDA-Approved Drugs for Combating Mycobacterium Abscessus and Mycobacterium chelonae. J. Antimicrob. Chemother. 66, 1533-1536. doi:10.1093/jac/dkr154
Christianson, S., Grierson, W., Kein, D., Tyler, A. D., Wolfe, J., and Sharma, M. K. (2016). Time-to-detection of Inducible Macrolide Resistance in mycobacterium Abscessus Subspecies and its Association with the Erm(41) Sequevar. PLoS One 11, e0158723. doi:10.1371/journal.pone.0158723

Čipčić Paljetak, H., Verbanac, D., Padovan, J., Dominis-Kramarić, M., Kelnerić, Ž., Perić, M., et al. (2016). Macrolones Are a Novel Class of Macrolide Antibiotics Active against Key Resistant Respiratory Pathogens In Vitro and In Vivo. Antimicrob. Agents Chemother. 60, 5337-5348. doi:10.1128/AAC.00524-16

Clark, C., Smith, K., Ednie, L., Bogdanovich, T., Dewasse, B., McGhee, P., et al. (2008). In Vitro activity of DC-159a, a New Broad-Spectrum Fluoroquinolone, Compared with that of Other Agents against Drug-Susceptible and -resistant Pneumococci. Antimicrob. Agents Chemother. 52, 77-84. doi:10.1128/ AAC. $01229-07$

Crunkhorn, S. (2018). Gallium Fights Infection in Phase I Trial. Nat. Rev. Drug Discov. 17, 786. doi:10.1038/nrd.2018.186

Cynamon, M., Jureller, J., Desai, B., Ramachandran, K., Sklaney, M., and Grossman, T. H. (2012). In Vitro activity of TP-271 against Mycobacterium Abscessus, Mycobacterium Fortuitum, and Nocardia Species. Antimicrob. Agents Chemother. 56, 3986-3988. doi:10.1128/AAC.00743-12

Daley, C. L., Iaccarino, J. M., Lange, C., Cambau, E., Wallace, R. J., Andrejak, C., et al. (2020). Treatment of Nontuberculous Mycobacterial Pulmonary Disease: An Official ATS/ERS/ESCMID/IDSA Clinical Practice Guideline. Eur. Respir. J. 56, 2000535. doi:10.1183/13993003.00535-2020

Daniel-Wayman, S., Shallom, S., Azeem, N., Olivier, K. N., Zelazny, A. M., and Prevots, D. R. (2019). Amikacin Exposure and Susceptibility of MacrolideResistant Mycobacterium Abscessus. ERJ Open Res. 5, 00154-02018. doi:10.1183/23120541.00154-2018

de Carvalho, N. F. G., Pavan, F., Sato, D. N., Leite, C. Q. F., Arbeit, R. D., and Chimara, E. (2018). Genetic Correlates of Clarithromycin Susceptibility Among Isolates of the Mycobacterium Abscessus Group and the Potential Clinical Applicability of a PCR-Based Analysis of Erm(41). J. Antimicrob. Chemother. 73, 862-866. doi:10.1093/jac/dkx476

De Groote, M. A., Jarvis, T. C., Wong, C., Graham, J., Hoang, T., Young, C. L., et al. (2018). Optimization and lead Selection of Benzothiazole Amide Analogs toward a Novel Antimycobacterial Agent. Front. Microbiol. 9, 2231. doi:10.3389/fmicb.2018.02231

Degiacomi, G., Benjak, A., Madacki, J., Boldrin, F., Provvedi, R., Palù, G., et al. (2017). Essentiality of mmpL3 and Impact of its Silencing on Mycobacterium tuberculosis Gene Expression. Sci. Rep. 7, 1-8. doi:10.1038/srep43495

Degiacomi, G., Sammartino, J. C., Chiarelli, L. R., Riabova, O., Makarov, V., and Pasca, M. R. (2019). Mycobacterium Abscessus, an Emerging and Worrisome Pathogen Among Cystic Fibrosis Patients. Ijms 20, 5868. doi:10.3390/ ijms 20235868

Dick, T., Shin, S. J., Koh, W.-J., Dartois, V., and Gengenbacher, M. (2020). Rifabutin Is Active against mycobacterium Abscessus in Mice. Antimicrob. Agents Chemother. 64, e01943-19. doi:10.1128/AAC.01943-19

Disratthakit, A., and Doi, N. (2010). In Vitro activities of DC-159a, a Novel Fluoroquinolone, against mycobacterium Species. Antimicrob. Agents Chemother. 54, 2684-2686. doi:10.1128/AAC.01545-09

Dubée, V., Bernut, A., Cortes, M., Lesne, T., Dorchene, D., Lefebvre, A.-L., et al. (2014). - Lactamase Inhibition by Avibactam in Mycobacterium Abscessus. J. Antimicrob. Chemother. 70, 1051-1058. doi:10.1093/jac/dku510

Dupont, C., Viljoen, A., Dubar, F., Blaise, M., Bernut, A., Pawlik, A., et al. (2016). A New Piperidinol Derivative Targeting Mycolic Acid Transport inMycobacterium Abscessus. Mol. Microbiol. 101, 515-529. doi:10.1111/ mmi.13406

Dupont, C., Viljoen, A., Thomas, S., Roquet-Banères, F., Herrmann, J.-L., Pethe, K., et al. (2017). Bedaquiline Inhibits the ATP Synthase in mycobacterium Abscessus and Is Effective in Infected Zebrafish. Antimicrob. Agents Chemother. 61, e01225-17. doi:10.1128/AAC.01225-17

Eckburg, P. B., Lister, T., Walpole, S., Keutzer, T., Utley, L., Tomayko, J., et al. (2019). Safety, Tolerability, Pharmacokinetics, and Drug Interaction Potential of SPR741, an Intravenous Potentiator, after Single and Multiple Ascending Doses and when Combined with $\beta$-Lactam Antibiotics in Healthy Subjects. Antimicrob. Agents Chemother. 63, e00892-19. doi:10.1128/AAC.00892-19

Falkinham, J. (2013). Ecology of Nontuberculous Mycobacteria-Where Do Human Infections Come from? Semin. Respir. Crit. Care Med. 34, 095-102. doi:10.1055/ s-0033-1333568 
Franz, N. D., Belardinelli, J. M., Kaminski, M. A., Dunn, L. C., Calado Nogueira de Moura, V., Blaha, M. A., et al. (2017). Design, Synthesis and Evaluation of Indole-2-Carboxamides with pan Anti-mycobacterial Activity. Bioorg. Med. Chem. 25, 3746-3755. doi:10.1016/j.bmc.2017.05.015

Ganapathy, U. S., Dartois, V., and Dick, T. (2019). Repositioning Rifamycins for Mycobacterium Abscessus Lung Disease. Expert Opin. Drug Discov. 14, 867-878. doi:10.1080/17460441.2019.1629414

Ganapathy, U. S., González del Rio, R., Cacho-Izquierdo, M., Ortega, F., Lelièvre, J., Barros-Aguirre, D., et al. (2021). A Leucyl-tRNA Synthetase Inhibitor with Broad-Spectrum Antimycobacterial Activity. Antimicrob. Agents Chemother. 65, e02420-20. doi:10.1128/aac.02420-20

Gebert, M. J., Delgado-Baquerizo, M., Oliverio, A. M., Webster, T. M., Nichols, L. M., Honda, J. R., et al. (2018). Ecological Analyses of Mycobacteria in Showerhead Biofilms and Their Relevance to Human Health. MBio 9, e01614-18. doi:10.1128/mbio.01614-18

Goldbart, A., Golan-Tripto, I., Pillar, G., Livnat-Levanon, G., Efrati, O., Spiegel, R., et al. (2020). Inhaled Nitric Oxide Therapy in Acute Bronchiolitis: A Multicenter Randomized Clinical Trial. Sci. Rep. 10, 9605. doi:10.1038/ s41598-020-66433-8

Goss, C. H., Kaneko, Y., Khuu, L., Anderson, G. D., Ravishankar, S., Aitken, M. L., et al. (2018). Gallium Disrupts Bacterial Iron Metabolism and Has Therapeutic Effects in Mice and Humans with Lung Infections. Sci. Transl. Med. 10, eaat7520. doi:10.1126/scitranslmed.aat7520

Gotfried, M. H., Horn, K., Garrity-Ryan, L., Villano, S., Tzanis, E., Chitra, S., et al. (2017). Comparison of Omadacycline and Tigecycline Pharmacokinetics in the Plasma, Epithelial Lining Fluid, and Alveolar Cells of Healthy Adult Subjects. Antimicrob. Agents Chemother. 61, e01135-17. doi:10.1128/AAC.01135-17

Grasemann, H., Michler, E., Wallot, M., and Ratjen, F. (1997). Decreased Concentration of Exhaled Nitric Oxide (NO) in Patients with Cystic Fibrosis. Pediatr. Pulmonol. 24, 173-177. doi:10.1002/(sici)10990496(199709)24:3<173::aid-ppul2>3.0.co;2-o

Greer, N. D. (2006). Tigecycline (Tygacil): The First in the Glycylcycline Class of Antibiotics. Baylor Univ. Med. Cent. Proc. 19, 155-161. doi:10.1080/ 08998280.2006.11928154

Griffith, D. E., Aksamit, T., Brown-Elliott, B. A., Catanzaro, A., Daley, C., Gordin, F., et al. (2007). An Official ATS/IDSA Statement: Diagnosis, Treatment, and Prevention of Nontuberculous Mycobacterial Diseases. Am. J. Respir. Crit. Care Med. 175, 367-416. doi:10.1164/rccm.200604-571ST

Grossman, T. H., Anderson, M. S., Christ, D., Gooldy, M., Henning, L. N., Heine, H. S., et al. (2017a). The Fluorocycline TP-271 Is Efficacious in Models of Aerosolized Francisella Tularensis SCHU S4 Infection in BALB/c Mice and Cynomolgus Macaques. Antimicrob. Agents Chemother. 61, e00448-17. doi:10.1128/AAC.00448-17

Grossman, T. H., Anderson, M. S., Drabek, L., Gooldy, M., Heine, H. S., Henning, L. N., et al. (2017b). The Fluorocycline TP-271 Is Efficacious in Models of Aerosolized Bacillus Anthracis Infection in BALB/c Mice and Cynomolgus Macaques. Antimicrob. Agents Chemother. 61, e01103-17. doi:10.1128/AAC.01103-17

Gupta, R., Netherton, M., Byrd, T. F., and Rohde, K. H. (2017). Reporter-based Assays for High-Throughput Drug Screening against Mycobacterium Abscessus. Front. Microbiol. 8, 2204. doi:10.3389/fmicb.2017.02204

Hanh, B. T. B., Kim, T. H., Park, J.-W., Lee, D.-G., Kim, J.-S., Du, Y. E., et al. (2020a). Etamycin as a Novel mycobacterium Abscessus Inhibitor. Ijms 21, 6908. doi:10.3390/ijms21186908

Hanh, B. T. B., Park, J.-W., Kim, T. H., Kim, J.-S., Yang, C.-S., Jang, K., et al. (2020b). Rifamycin O, an Alternative Anti-Mycobacterium Abscessus Agent. Molecules 25, 1597. doi:10.3390/molecules25071597

Harada, T., Akiyama, Y., Kurashima, A., Nagai, H., Tsuyuguchi, K., Fujii, T., et al. (2012). Clinical and Microbiological Differences between Mycobacterium Abscessus and Mycobacterium Massiliense Lung Diseases. J. Clin. Microbiol. 50, 3556-3561. doi:10.1128/JCM.01175-12

Hartmann, R., Höltje, J.-V., and Schwarz, U. (1972). Targets of Penicillin Action in Escherichia coli. Nature 235, 426-429. doi:10.1038/235426a0

Hatakeyama, S., Ohama, Y., Okazaki, M., Nukui, Y., and Moriya, K. (2017). Antimicrobial Susceptibility Testing of Rapidly Growing Mycobacteria Isolated in Japan. BMC Infect. Dis. 17, 197. doi:10.1186/s12879-017-2298-8

Haworth, C. S., Banks, J., Capstick, T., Fisher, A. J., Gorsuch, T., Laurenson, I. F., et al. (2017). British Thoracic Society Guidelines for the Management of Non-tuberculous
Mycobacterial Pulmonary Disease (NTM-PD). Thorax 72, iil-ii64. doi:10.1136/ thoraxjnl-2017-210927

Ho, L. P., Innes, J. A., and Greening, A. P. (1998). Exhaled Nitric Oxide Is Not Elevated in the Inflammatory Airways Diseases of Cystic Fibrosis and Bronchiectasis. Eur. Respir. J. 12, 1290-1294. doi:10.1183/09031936.98.12061290

Hoshino, K., Inoue, K., Murakami, Y., Kurosaka, Y., Namba, K., Kashimoto, Y., et al. (2008). In Vitro and In Vivo Antibacterial Activities of DC-159a, a New Fluoroquinolone. Antimicrob. Agents Chemother. 52, 65-76. doi:10.1128/ AAC.00853-07

Jelić, D., and Antolović, R. (2016). From Erythromycin to Azithromycin and New Potential Ribosome-Binding Antimicrobials. Antibiotics (Basel) 5, 29. doi:10.3390/antibiotics5030029

Jeon, K., Kwon, O. J., Lee, N. Y., Kim, B.-J., Kook, Y.-H., Lee, S.-H., et al. (2009). Antibiotic Treatment ofMycobacterium abscessusLung Disease. Am. J. Respir. Crit. Care Med. 180, 896-902. doi:10.1164/rccm.200905-0704OC

Jeong, J., Kim, G., Moon, C., Kim, H. J., Kim, T. H., and Jang, J. (2018). Pathogen Box Screening for Hit Identification against Mycobacterium Abscessus. PLoS One 13, e0195595. doi:10.1371/journal.pone.0195595

Jeong, S. H., Kim, S.-Y., Huh, H. J., Ki, C.-S., Lee, N. Y., Kang, C.-I., et al. (2017). Mycobacteriological Characteristics and Treatment Outcomes in Extrapulmonary Mycobacterium Abscessus Complex Infections. Int. J. Infect. Dis. 60, 49-56. doi:10.1016/j.ijid.2017.05.007

Johansen, M. D., Daher, W., Roquet-Banères, F., Raynaud, C., Alcaraz, M., Maurer, F. P., et al. (2020a). Rifabutin Is Bactericidal against Intracellular and Extracellular Forms of mycobacterium Abscessus. Antimicrob. Agents Chemother. 64, e00363-20. doi:10.1128/AAC.00363-20

Johansen, M. D., Herrmann, J.-L., and Kremer, L. (2020b). Non-tuberculous Mycobacteria and the Rise of Mycobacterium Abscessus. Nat. Rev. Microbiol. 18, 392-407. doi:10.1038/s41579-020-0331-1

Just-Baringo, X., Albericio, F., and Álvarez, M. (2014). Thiopeptide Antibiotics: Retrospective and Recent Advances. Mar. Drugs 12, 317-351. doi:10.3390/ md12010317

Kaushik, A., Ammerman, N. C., Martins, O., Parrish, N. M., and Nuermberger, E. L. (2019). In Vitro activity of New Tetracycline Analogs Omadacycline and Eravacycline against Drug-Resistant Clinical Isolates of mycobacterium Abscessus. Antimicrob. Agents Chemother. 63, e00470-19. doi:10.1128/AAC.00470-19

Kaushik, A., Gupta, C., Fisher, S., Story-Roller, E., Galanis, C., Parrish, N., et al. (2017). Combinations of Avibactam and Carbapenems Exhibit Enhanced Potencies against Drug-Resistant Mycobacterium Abscessus. Future Microbiol. 12, 473-480. doi:10.2217/fmb-2016-0234

Keen, C., Gustafsson, P., Lindblad, A., Wennergren, G., and Olin, A.-C. (2010). Low Levels of Exhaled Nitric Oxide Are Associated with Impaired Lung Function in Cystic Fibrosis. Pediatr. Pulmonol. 45, 241-248. doi:10.1002/ppul.21137

Kim, S.-Y., Jhun, B. W., Moon, S. M., Shin, S. H., Jeon, K., Kwon, O. J., et al. (2018). Mutations in gyrA and gyrB in Moxifloxacin-Resistant mycobacterium Avium Complex and mycobacterium Abscessus Complex Clinical Isolates. Antimicrob. Agents Chemother. 62, e00527-18. doi:10.1128/AAC.00527-18

Kim, T., Hanh, B.-T. -B., Heo, B., Quang, N., Park, Y., Shin, J., et al. (2021). A Screening of the MMV Pandemic Response Box Reveals Epetraborole as A New Potent Inhibitor against Mycobacterium Abscessus. Ijms 22, 5936. doi:10.3390/ ijms22115936

Kim, T. H., Hanh, B. T. B., Kim, G., Lee, D.-G., Park, J.-W., Lee, S. E., et al. (2019). Thiostrepton: A Novel Therapeutic Drug Candidate for Mycobacterium Abscessus Infection. Molecules 24, 4511. doi:10.3390/molecules24244511

Kim, T. S., Choe, J. H., Kim, Y. J., Yang, C.-S., Kwon, H.-J., Jeong, J., et al. (2017). Activity of LCB01-0371, a Novel Oxazolidinone, against mycobacterium Abscessus. Antimicrob. Agents Chemother. 61, e02752-16. doi:10.1128/ AAC.02752-16

Koh, W.-J., Jeon, K., Lee, N. Y., Kim, B.-J., Kook, Y.-H., Lee, S.-H., et al. (2011). Clinical Significance of Differentiation ofMycobacterium massiliensefromMycobacterium Abscessus. Am. J. Respir. Crit. Care Med. 183, 405-410. doi:10.1164/rccm.201003-0395OC

Koh, W.-J., Stout, J. E., and Yew, W.-W. (2014). Advances in the Management of Pulmonary Disease Due to Mycobacterium Abscessus Complex. Int. J. Tuberc. Lung Dis. 18, 1141-1148. doi:10.5588/ijtld.14.0134

Koul, A., Dendouga, N., Vergauwen, K., Molenberghs, B., Vranckx, L., Willebrords, R., et al. (2007). Diarylquinolines Target Subunit C of Mycobacterial ATP Synthase. Nat. Chem. Biol. 3, 323-324. doi:10.1038/nchembio884 
Kozikowski, A. P., Onajole, O. K., Stec, J., Dupont, C., Viljoen, A., Richard, M., et al. (2017). Targeting Mycolic Acid Transport by Indole-2-Carboxamides for the Treatment of Mycobacterium Abscessus Infections. J. Med. Chem. 60, 5876-5888. doi:10.1021/acs.jmedchem.7b00582

Lavollay, M., Fourgeaud, M., Herrmann, J.-L., Dubost, L., Marie, A., Gutmann, L., et al. (2011). The Peptidoglycan of Mycobacterium Abscessus Is Predominantly Cross-Linked by L, D -Transpeptidases. J. Bacteriol. 193, 778-782. doi:10.1128/ JB.00606-10

Le Run, E., Arthur, M., and Mainardi, J.-L. (2018). In Vitro and Intracellular Activity of Imipenem Combined with Rifabutin and Avibactam against mycobacterium Abscessus. Antimicrob. Agents Chemother. 62, e00623-18. doi:10.1128/AAC.00623-18

Lee, H., Sohn, Y. M., Ko, J. Y., Lee, S.-Y., Jhun, B. W., Park, H. Y., et al. (2017). Once-daily Dosing of Amikacin for Treatment of Mycobacterium Abscessus Lung Disease. Int. J. Tuberc. Lung Dis. 21, 818-824. doi:10.5588/ijtld.16.0791

Lee, Y. R., and Burton, C. E. (2019). Eravacycline, a Newly Approved Fluorocycline. Eur. J. Clin. Microbiol. Infect. Dis. 38, 1787-1794. doi:10.1007/s10096-019-03590-3

Lefebvre, A.-L., Le Moigne, V., Bernut, A., Veckerlé, C., Compain, F., Herrmann, J.L., et al. (2017). Inhibition of the $\beta$-Lactamase Bla Mab by Avibactam Improves the In Vitro and In Vivo Efficacy of Imipenem against Mycobacterium Abscessus. Antimicrob. Agents Chemother. 61, e02440-16. doi:10.1128/ aac.02440-16

Lerat, I., Cambau, E., Roth Dit Bettoni, R., Gaillard, J.-L., Jarlier, V., Truffot, C., et al. (2014). In Vivo evaluation of Antibiotic Activity against Mycobacterium Abscessus. J. Infect. Dis. 209, 905-912. doi:10.1093/infdis/jit614

Li, W., Yazidi, A., Pandya, A. N., Hegde, P., Tong, W., Calado Nogueira de Moura, V., et al. (2018). MmpL3 as a Target for the Treatment of Drug-Resistant Nontuberculous Mycobacterial Infections. Front. Microbiol. 9, 1547. doi:10.3389/fmicb.2018.01547

Lipman, M., Kunst, H., Loebinger, M. R., Milburn, H. J., and King, M. (2021). Non Tuberculous Mycobacteria Pulmonary Disease: Patients and Clinicians Working Together to Improve the Evidence Base for Care. Int. J. Infect. Dis., S1201-S9712. doi:10.1016/j.ijid.2021.03.064

Locher, C. P., Jones, S. M., Hanzelka, B. L., Perola, E., Shoen, C. M., Cynamon, M. H., et al. (2015). A Novel Inhibitor of Gyrase B Is a Potent Drug Candidate for Treatment of Tuberculosis and Nontuberculosis Mycobacterial Infections. Antimicrob. Agents Chemother. 59, 1455-1465. doi:10.1128/AAC.04347-14

Lyu, J., Jang, H. J., Song, J. W., Choi, C.-M., Oh, Y.-M., Lee, S. D., et al. (2011). Outcomes in Patients with Mycobacterium Abscessus Pulmonary Disease Treated with Long-Term Injectable Drugs. Respir. Med. 105, 781-787. doi:10.1016/j.rmed.2010.12.012

Maggioncalda, E. C., Story-Roller, E., Mylius, J., Illei, P., Basaraba, R. J., and Lamichhane, G. (2020). A Mouse Model of Pulmonary Mycobacteroides Abscessus Infection. Sci. Rep. 10, 3690. doi:10.1038/s41598-020-60452-1

Malin, J. J., Winter, S., Van Gumpel, E., Plum, G., and Rybniker, J. (2019). Extremely Low Hit Rate in a Diverse Chemical Drug Screen Targeting mycobacterium Abscessus. Antimicrob. Agents Chemother. 63, e01008-19. doi:10.1128/AAC.01008-19

Martiniano, S. L., Wagner, B. D., Levin, A., Nick, J. A., Sagel, S. D., Daley, C. L., et al. (2017). Safety and Effectiveness of Clofazimine for Primary and Refractory Nontuberculous Mycobacterial Infection. Chest 152, 800-809. doi:10.1016/ j.chest.2017.04.175

Maurer, F. P., Castelberg, C., Quiblier, C., Böttger, E. C., and Somoskövi, A. (2014). Erm(41)-dependent Inducible Resistance to Azithromycin and Clarithromycin in Clinical Isolates of mycobacterium Abscessus. J. Antimicrob. Chemother. 69, 1559-1563. doi:10.1093/jac/dku007

McGuffin, S. A., Pottinger, P. S., and Harnisch, J. P. (2017). Clofazimine in Nontuberculous Mycobacterial Infections: A Growing Niche. Open Forum Infect. Dis. 4, ofx147. doi:10.1093/ofid/ofx147

McNeil, M. B., O'Malley, T., Dennison, D., Shelton, C. D., Sunde, B., and Parish, T. (2020). Multiple Mutations in Mycobacterium tuberculosis MmpL3 Increase Resistance to MmpL3 Inhibitors. mSphere 5, 502-511. doi:10.1128/ msphere.00985-20

Minias, A., Żukowska, L., Lach, J., Jagielski, T., Strapagiel, D., Kim, S.-Y., et al. (2020). Subspecies-specific Sequence Detection for Differentiation of Mycobacterium Abscessus Complex. Sci. Rep. 10, 16415. doi:10.1038/ s41598-020-73607-x
Moigne, V. Le., Moreau, F., Dupont, C., Neyrolles, O., Kremer, L., and Herrmann, J. (2020). Efficacy of Bedaquiline, Alone or in Combination with Imipenem, against Mycobacterium Abscessus in $\mathrm{C} 3 \mathrm{HeB} / \mathrm{FeJ}$ Mice. Antimicrob. Agents Chemother. 64, e00114-20.

Morimoto, K., Aono, A., Murase, Y., Sekizuka, T., Kurashima, A., Takaki, A., et al. (2018). Prevention of Aerosol Isolation of Nontuberculous mycobacterium from the Patient's Bathroom. ERJ Open Res. 4, 00150-02017. doi:10.1183/ 23120541.00150-2017

Mougari, F., Guglielmetti, L., Raskine, L., Sermet-Gaudelus, I., Veziris, N., and Cambau, E. (2016). Infections Caused byMycobacterium Abscessus: Epidemiology, Diagnostic Tools and Treatment. Expert Rev. Anti-infective Ther. 14, 1139-1154. doi:10.1080/14787210.2016.1238304

Munić Kos, V., Koštrun, S., Fajdetić, A., Bosnar, M., Kelnerić, Ž., Stepanić, V., et al. (2013). Structure-property Relationship for Cellular Accumulation of Macrolones in Human Polymorphonuclear Leukocytes (PMNs). Eur. J. Pharm. Sci. 49, 206-219. doi:10.1016/j.ejps.2013.02.019

Nash, K. A., Brown-Elliott, B. A., and Wallace, R. J. (2009). A Novel Gene, Erm (41), Confers Inducible Macrolide Resistance to Clinical Isolates of Mycobacterium Abscessus but Is Absent from Mycobacterium chelonae. Antimicrob. Agents Chemother. 53, 1367-1376. doi:10.1128/AAC.01275-08

Nessar, R., Cambau, E., Reyrat, J. M., Murray, A., and Gicquel, B. (2012). Mycobacterium Abscessus: A New Antibiotic Nightmare. J. Antimicrob. Chemother. 67, 810-818. doi:10.1093/jac/dkr578

O’Dwyer, K., Spivak, A. T., Ingraham, K., Min, S., Holmes, D. J., Jakielaszek, C., et al. (2015). Bacterial Resistance to Leucyl-tRNA Synthetase Inhibitor GSK2251052 Develops during Treatment of Complicated Urinary Tract Infections. Antimicrob. Agents Chemother. 59, 289-298. doi:10.1128/AAC.03774-14

Obrecht, D., Bernardini, F., Dale, G., and Dembowsky, K. (2011). Emerging New Therapeutics against Key Gram-Negative Pathogens. Annu. Rep. Med. Chem., 245-262. doi:10.1016/B978-0-12-386009-5.00012-6

Obregón-Henao, A., Arnett, K. A., Henao-Tamayo, M., Massoudi, L., Creissen, E., Andries, K., et al. (2015). Susceptibility of mycobacterium Abscessus to Antimycobacterial Drugs in Preclinical Models. Antimicrob. Agents Chemother. 59, 6904-6912. doi:10.1128/AAC.00459-15

Oh, C.-T., Moon, C., Park, O. K., Kwon, S.-H., and Jang, J. (2014). Novel Drug Combination for Mycobacterium Abscessus Disease Therapy Identified in a Drosophila Infection Model. J. Antimicrob. Chemother. 69, 1599-1607. doi:10.1093/jac/dku024

Olaru, I. D., Von Groote-Bidlingmaier, F., Heyckendorf, J., Yew, W. W., Lange, C., and Chang, K. C. (2015). Novel Drugs against Tuberculosis: a Clinician's Perspective. Eur. Respir. J. 45, 1119-1131. doi:10.1183/ 09031936.00162314

Olivier, K. N., Griffith, D. E., Eagle, G., McGinnis, J. P., Micioni, L., Liu, K., et al. (2017). Randomized Trial of Liposomal Amikacin for Inhalation in Nontuberculous Mycobacterial Lung Disease. Am. J. Respir. Crit. Care Med. 195, 814-823. doi:10.1164/rccm.201604-0700OC

Palencia, A., Li, X., Bu, W., Choi, W., Ding, C. Z., Easom, E. E., et al. (2016). Discovery of Novel Oral Protein Synthesis Inhibitors of mycobacterium Tuberculosis that Target Leucyl-tRNA Synthetase. Antimicrob. Agents Chemother. 60, 6271-6280. doi:10.1128/AAC.01339-16

Pandey, R., Chen, L., Manca, C., Jenkins, S., Glaser, L., Vinnard, C., et al. (2019). Dual $\beta$-Lactam Combinations Highly Active against Mycobacterium Abscessus Complex In Vitro. MBio 10, e02895-18. doi:10.1128/mBio.02895-18

Pandya, A. N., Prathipati, P. K., Hegde, P., Li, W., Graham, K. F., Mandal, S., et al. (2019). Indole-2-carboxamides Are Active against mycobacterium Abscessus in a Mouse Model of Acute Infection. Antimicrob. Agents Chemother. 63, e02245-18. doi:10.1128/AAC.02245-18

Park, J., Cho, J., Lee, C.-H., Han, S. K., and Yim, J.-J. (2017). Progression and Treatment Outcomes of Lung Disease Caused by mycobacterium Abscessus and mycobacterium Massiliense. Clin. Infect. Dis. 64, 301-308. doi:10.1093/cid/ ciw723

Park, S., Kim, S., Park, E. M., Kim, H., Kwon, O. J., Chang, C. L., et al. (2008). In Vitro antimicrobial Susceptibility of Mycobacterium Abscessus in Korea. J. Korean Med. Sci. 23, 49-52. doi:10.3346/jkms.2008.23.1.49

Pearce, C., Ruth, M. M., Pennings, L. J., Wertheim, H. F. L., Walz, A., Hoefsloot, W., et al. (2020). Inhaled Tigecycline Is Effective against Mycobacterium Abscessus In Vitro and In Vivo. J. Antimicrob. Chemother. 75, 1889-1894. doi:10.1093/jac/dkaa110 
Pearson, J. C., Dionne, B., Richterman, A., Vidal, S. J., Weiss, Z., Velásquez, G. E., et al. (2020). Omadacycline for the Treatment of Mycobacterium Abscessus Disease: A Case Series. Open Forum Infect. Dis. 7, ofaa415. doi:10.1093/ofid/ofaa415

Philley, J. V., Wallace, R. J., Benwill, J. L., Taskar, V., Brown-Elliott, B. A., Thakkar, F., et al. (2015). Preliminary Results of Bedaquiline as Salvage Therapy for Patients with Nontuberculous Mycobacterial Lung Disease. Chest 148, 499-506. doi:10.1378/chest.14-2764

Pryjma, M., Burian, J., Kuchinski, K., and Thompson, C. J. (2017). Antagonism between Front-Line Antibiotics Clarithromycin and Amikacin in the Treatment of Mycobacterium Abscessus Infections Is Mediated by the whiB7 Gene. Antimicrob. Agents Chemother. 61, e01353-17. doi:10.1128/ AAC.01347-1710.1128/aac.01353-17

Purnapatre, K. P., Rao, M., Pandya, M., Khanna, A., Chaira, T., Bambal, R., et al. (2018). In Vitro and In Vivo Activities of DS86760016, a Novel Leucyl-tRNA Synthetase Inhibitor for Gram-Negative Pathogens. Antimicrob. Agents Chemother. 62, e01987-17. doi:10.1128/AAC.01987-17

Ratnatunga, C. N., Lutzky, V. P., Kupz, A., Doolan, D. L., Reid, D. W., Field, M., et al. (2020). The Rise of Non-tuberculosis Mycobacterial Lung Disease. Front. Immunol. 11, 303. doi:10.3389/fimmu.2020.00303

Raynaud, C., Daher, W., Johansen, M. D., Roquet-Banères, F., Blaise, M., Onajole, O. K., et al. (2020a). Active Benzimidazole Derivatives Targeting the MmpL3 Transporter in Mycobacterium Abscessus. ACS Infect. Dis. 6, 324-337. doi:10.1021/acsinfecdis.9b00389

Raynaud, C., Daher, W., Roquet-Banères, F., Johansen, M. D., Stec, J., Onajole, O. K., et al. (2020b). Synergistic Interactions of Indole-2-Carboxamides and $\beta$-Lactam Antibiotics against Mycobacterium Abscessus. Antimicrob. Agents Chemother. 64, e02548-19. doi:10.1128/AAC.02548-19

Richard, M., Gutiérrez, A. V., and Kremer, L. (2020). Dissecting Erm (41)Mediated Macrolide-Inducible Resistance in Mycobacterium Abscessus. Antimicrob. Agents Chemother. 64, e01879-19. doi:10.1128/AAC.01879-19

Richter, A., Strauch, A., Chao, J., Ko, M., and Av-Gay, Y. (2018). Screening of Preselected Libraries Targeting mycobacterium Abscessus for Drug Discovery. Antimicrob. Agents Chemother. 62, e00828-18. doi:10.1128/AAC.00828-18

Rominski, A., Roditscheff, A., Selchow, P., Böttger, E. C., and Sander, P. (2017). Intrinsic Rifamycin Resistance ofMycobacterium Abscessusis Mediated by ADP-Ribosyltransferase MAB_0591. J. Antimicrob. Chemother. 72, 376-384. doi:10.1093/jac/dkw466

Rosati, M. (2017). Saunders Handbook of Veterinary Drugs: Small and Large Animals. Can. Vet. J. 4th edition, 451-452.

Rose, S. J., Neville, M. E., Gupta, R., and Bermudez, L. E. (2014). Delivery of Aerosolized Liposomal Amikacin as a Novel Approach for the Treatment of Nontuberculous Mycobacteria in an Experimental Model of Pulmonary Infection. PLoS One 9, e108703. doi:10.1371/journal.pone.0108703

Roux, A.-L., Viljoen, A., Bah, A., Simeone, R., Bernut, A., Laencina, L., et al. (2016). The Distinct Fate of Smooth and Rough Mycobacterium Abscessus Variants inside Macrophages. Open Biol. 6, 160185. doi:10.1098/rsob.160185

Rubio, A., Stapleton, M., and Verman, D. (2018). Potent Activity of a Novel Gyrase Inhibitor (SPR719/720) in Vitro and in a Prolonged Acute Mycobacterium Abscessus Mouse Model of Infection. Atlanta, GA: ASM Microbe.

Rubio, M., March, F., Garrigó, M., Moreno, C., Español, M., and Coll, P. (2015). Inducible and Acquired Clarithromycin Resistance in the mycobacterium Abscessus Complex. PLoS One 10, e0140166. doi:10.1371/journal.pone.0140166

Rudra, P., Hurst-Hess, K., Lappierre, P., and Ghosh, P. (2018). High Levels of Intrinsic Tetracycline Resistance in mycobacterium Abscessus Are Conferred by a Tetracycline-Modifying Monooxygenase. Antimicrob. Agents Chemother. 62, e00119-18. doi:10.1128/AAC.00119-18

Ryan, K., and Byrd, T. F. (2018). Mycobacterium Abscessus: Shapeshifter of the Mycobacterial World. Front. Microbiol. 9, 2642. doi:10.3389/fmicb.2018.02642

Sabin, A. P., Ferrieri, P., and Kline, S. (2017). Mycobacterium Abscessus Complex Infections in Children: A Review. Curr. Infect. Dis. Rep. 19, 1-12. doi:10.1007/ s11908-017-0597-2

Sarathy, J. P., Ganapathy, U. S., Zimmerman, M. D., Dartois, V., Gengenbacher, M., and Dick, T. (2020). TBAJ-876, a 3,5-Dialkoxypyridine Analogue of Bedaquiline, Is Active against Mycobacterium Abscessus. Antimicrob. Agents Chemother. 64, e02404-19. doi:10.1128/AAC.02404-19

Sassi, M., and Drancourt, M. (2014). Genome Analysis Reveals Three Genomospecies in Mycobacterium Abscessus. BMC Genomics 15, 359. doi:10.1186/1471-2164-15-359
Schäfle, D., Selchow, P., Borer, B., Meuli, M., Rominski, A., Schulthess, B., et al. (2021). Rifabutin Is Inactivated by mycobacterium Abscessus Arr. Antimicrob. Agents Chemother. 65, 4-7. doi:10.1128/AAC.02215-20

Sensi, P., Margalith, P., and Timbal, M. T. (1959). Rifomycin, a New Antibiotic; Preliminary Report. Farmaco. Sci. 14, 146-147.

Shallom, S. J., Gardina, P. J., Myers, T. G., Sebastian, Y., Conville, P., Calhoun, L. B., et al. (2013). New Rapid Scheme for Distinguishing the Subspecies of the Mycobacterium Abscessus Group and Identifying Mycobacterium Massiliense Isolates with Inducible Clarithromycin Resistance. J. Clin. Microbiol. 51, 2943-2949. doi:10.1128/JCM.01132-13

Shen, G.-H., Wu, B.-D., Hu, S.-T., Lin, C.-F., Wu, K.-M., and Chen, J.-H. (2010). High Efficacy of Clofazimine and its Synergistic Effect with Amikacin against Rapidly Growing Mycobacteria. Int. J. Antimicrob. Agents 35, 400-404. doi:10.1016/j.ijantimicag.2009.12.008

Shi, K., Caldwell, S. J., Fong, D. H., and Berghuis, A. M. (2013). Prospects for Circumventing Aminoglycoside Kinase Mediated Antibiotic Resistance. Front. Cel. Infect. Microbiol. 3, 1-17. doi:10.3389/fcimb.2013.00022

Shin, S. J., Choi, G.-E., Cho, S.-N., Woo, S. Y., Jeong, B.-H., Jeon, K., et al. (2013). Mycobacterial Genotypes Are Associated with Clinical Manifestation and Progression of Lung Disease Caused by mycobacterium Abscessus and mycobacterium Massiliense. Clin. Infect. Dis. 57, 32-39. doi:10.1093/cid/cit172

Silva, C. (2020). Molgradex Stops Bacteria in Bronchiectasis Patients with Lung Infections, Trial Shows. Bronchiectasis News Today. Available at: https:// bronchiectasisnewstoday.com/2020/03/16/molgradex-stops-bacterial-growth-inbronchiectasis-patients-with-chronic-lung-infection-phase-2a-trial-shows/.

Singh, S., Bouzinbi, N., Chaturvedi, V., Godreuil, S., and Kremer, L. (2014). In Vitro evaluation of a New Drug Combination against Clinical Isolates Belonging to the Mycobacterium Abscessus Complex. Clin. Microbiol. Infect. 20, O1124-O1127. doi:10.1111/1469-0691.12780

Skolnik, K., Kirkpatrick, G., and Quon, B. S. (2016). Nontuberculous Mycobacteria in Cystic Fibrosis. Curr. Treat. Options. Infect. Dis. 8, 259-274. doi:10.1007/ s40506-016-0092-6

Soni, I., De Groote, M. A., Dasgupta, A., and Chopra, S. (2016). Challenges Facing the Drug Discovery Pipeline for Non-tuberculous Mycobacteria. J. Med. Microbiol. 65, 1-8. doi:10.1099/jmm.0.000198

Sorayah, R., Manimekalai, M. S. S., Shin, S. J., Koh, W.-J., Grüber, G., and Pethe, K. (2019). Naturally-Occurring Polymorphisms in QcrB Are Responsible for Resistance to Telacebec in Mycobacterium Abscessus. ACS Infect. Dis. 5, 2055-2060. doi:10.1021/acsinfecdis.9b00322

Soroka, D., Dubée, V., Soulier-Escrihuela, O., Cuinet, G., Hugonnet, J.-E., Gutmann, L., et al. (2014). Characterization of Broad-Spectrum Mycobacterium Abscessus Class A -lactamase. J. Antimicrob. Chemother. 69, 691-696. doi:10.1093/jac/dkt410

Stephenson, D., Perry, A., Appleby, M. R., Lee, D., Davison, J., Johnston, A., et al. (2019). An Evaluation of Methods for the Isolation of Nontuberculous Mycobacteria from Patients with Cystic Fibrosis, Bronchiectasis and Patients Assessed for Lung Transplantation. BMC Pulm. Med. 19, 19. doi:10.1186/ s12890-019-0781-2

Story-Roller, E., Maggioncalda, E. C., and Lamichhane, G. (2019). Select $\beta$-Lactam Combinations Exhibit Synergy against Mycobacterium Abscessus In Vitro. Antimicrob. Agents Chemother. 63, e02613-18. doi:10.1128/AAC.02613-18

Stout, J. E., and Floto, R. A. (2012). Treatment ofMycobacterium Abscessus. Am. J. Respir. Crit. Care Med. 186, 822-823. doi:10.1164/rccm.201208-1500ED

Tang, Y. W., Cheng, B., Yeoh, S. F., Lin, R. T. P., and Teo, J. W. P. (2018). Tedizolid Activity against Clinical Mycobacterium Abscessus Complex Isolates-An In Vitro Characterization Study. Front. Microbiol. 9, 2095. doi:10.3389/ fmicb.2018.02095

Trzasko, A., Leeds, J. A., Praestgaard, J., LaMarche, M. J., and McKenney, D. (2012). Efficacy of LFF571 in a Hamster Model of Clostridium difficile Infection. Antimicrob. Agents Chemother. 56, 4459-4462. doi:10.1128/ AAC.06355-11

Verma, D., Peterson, C., Cotroneo, N. S., Stokes, S., and Ordway, D. J. (2020). 1637. SPR720, A Novel Benzamidazole Gyrase Inhibitor, Demonstrates Potent Efficacy against Mycobacterium avium ATCC 700898 in a Chronic C3HeBFeJ Mouse Infection Model. Open Forum Infect. Dis. 7, S809. doi:10.1093/ofid/ofaa439.1817

Verma, M., Kaur, J., Kumar, M., Kumari, K., Saxena, A., Anand, S., et al. (2011). Whole Genome Sequence of the Rifamycin B-Producing Strain Amycolatopsis Mediterranei S699. J. Bacteriol. 193, 5562-5563. doi:10.1128/JB.05819-11 
Victoria, L., Gupta, A., Gómez, J. L., and Robledo, J. (2021). Mycobacterium Abscessus Complex: A Review of Recent Developments in an Emerging Pathogen. Front. Cel. Infect. Microbiol. 11, 1-8. doi:10.3389/ fcimb.2021.659997

Viljoen, A., Raynaud, C., Johansen, M. D., Roquet-Banères, F., Herrmann, J.-L., Daher, W., et al. (2019). Verapamil Improves the Activity of Bedaquiline against Mycobacterium abscessusIn Vitro and in Macrophages. Antimicrob. Agents Chemother. 63, e00705-19. doi:10.1128/AAC.00705-19

Wallace, R. J., Brown-Elliott, B. A., Ward, S. C., Crist, C. J., Mann, L. B., and Wilson, R. W. (2001). Activities of Linezolid against Rapidly Growing Mycobacteria. Antimicrob. Agents Chemother. 45, 764-767. doi:10.1128/ AAC.45.3.764-767.2001

Wallace, R. J., Dukart, G., Brown-Elliott, B. A., Griffith, D. E., Scerpella, E. G., and Marshall, B. (2014). Clinical Experience in 52 Patients with TigecyclineContaining Regimens for Salvage Treatment of Mycobacterium Abscessus and Mycobacterium chelonae Infections. J. Antimicrob. Chemother. 69, 1945-1953. doi:10.1093/jac/dku062

Warrell, R. P., Skelos, A., Alcock, N. W., and Bockman, R. S. (1986). Gallium Nitrate for Acute Treatment of Cancer-Related Hypercalcemia: Clinicopharmacological and Dose Response Analysis. Cancer Res. 46, 4208-4212.

Wassilew, N., Hoffmann, H., Andrejak, C., and Lange, C. (2016). Pulmonary Disease Caused by Non-tuberculous Mycobacteria. Respiration 91, 386-402. doi: $10.1159 / 000445906$

Wen, S., Gao, X., Zhao, W., Huo, F., Jiang, G., Dong, L., et al. (2021). In Vitro efficacy Comparison of Linezolid, Tedizolid, Sutezolid and Delpazolid against Rapid Growing Mycobacteria Isolated in Beijing, China. Int. J. Infect. Dis., S1201-S9712. doi:10.1101/2020.06.25.172742

Wi, Y. M. (2019). Treatment of Extrapulmonary Nontuberculous Mycobacterial Diseases. Infect. Chemother. 51, 245-255. doi:10.3947/ic.2019.51.3.245

Wu, M.-L., Aziz, D. B., Dartois, V., and Dick, T. (2018). NTM Drug Discovery: Status, Gaps and the Way Forward. Drug Discov. TodayToday 23, 1502-1519. doi:10.1016/j.drudis.2018.04.001

Xue, Q., Yan, Y., Zhang, R., and Xiong, H. (2018). Regulation of iNOS on Immune Cells and its Role in Diseases. Ijms 19, 3805. doi:10.3390/ ijms19123805
Yaacoby-Bianu, K., Gur, M., Toukan, Y., Nir, V., Hakim, F., Geffen, Y., et al. (2018) Compassionate Nitric Oxide Adjuvant Treatment of Persistent Mycobacterium Infection in Cystic Fibrosis Patients. Pediatr. Infect. Dis. J. 37, 336-338. doi:10.1097/INF.0000000000001780

Yang, B., Jhun, B. W., Moon, S. M., Lee, H., Park, H. Y., Jeon, K., et al. (2017). Clofazimine-containing Regimen for the Treatment of mycobacterium Abscessus Lung Disease. Antimicrob. Agents Chemother. 61, e02052-16. doi:10.1128/AAC.02052-16

Yano, T., Kassovska-Bratinova, S., Teh, J. S., Winkler, J., Sullivan, K., Isaacs, A., et al. (2011). Reduction of Clofazimine by Mycobacterial Type 2 NADH: Quinone Oxidoreductase. J. Biol. Chem. 286, 10276-10287. doi:10.1074/ jbc.M110.200501

Zhang, J., Leifer, F., Rose, S., Chun, D. Y., Thaisz, J., Herr, T., et al. (2018). Amikacin Liposome Inhalation Suspension (ALIS) Penetrates Non-tuberculous Mycobacterial Biofilms and Enhances Amikacin Uptake into Macrophages. Front. Microbiol. 9, 915. doi:10.3389/fmicb.2018.00915

Zhang, S., Zou, Y., Guo, Q., Chen, J., Xu, L., Wan, X., et al. (2020). AR-12 Exhibits Direct and Host-Targeted Antibacterial Activity toward mycobacterium Abscessus. Antimicrob. Agents Chemother. 64, e00236-20. doi:10.1128/AAC.00236-20

Conflict of Interest: The authors declare that the research was conducted in the absence of any commercial or financial relationships that could be construed as a potential conflict of interest.

Publisher's Note: All claims expressed in this article are solely those of the authors and do not necessarily represent those of their affiliated organizations, or those of the publisher, the editors and the reviewers. Any product that may be evaluated in this article, or claim that may be made by its manufacturer, is not guaranteed or endorsed by the publisher.

Copyright (c) 2021 Quang and Jang. This is an open-access article distributed under the terms of the Creative Commons Attribution License (CC BY). The use, distribution or reproduction in other forums is permitted, provided the original author(s) and the copyright owner(s) are credited and that the original publication in this journal is cited, in accordance with accepted academic practice. No use, distribution or reproduction is permitted which does not comply with these terms. 